\title{
Wave turbulence and intermittency
}

\author{
Alan C. Newell*,1, Sergey Nazarenko, Laura Biven \\ Department of Mathematics, University of Warwick, Coventry CV4 71L, UK
}

\begin{abstract}
In the early 1960s, it was established that the stochastic initial value problem for weakly coupled wave systems has a natural asymptotic closure induced by the dispersive properties of the waves and the large separation of linear and nonlinear time scales. One is thereby led to kinetic equations for the redistribution of spectral densities via three- and four-wave resonances together with a nonlinear renormalization of the frequency. The kinetic equations have equilibrium solutions which are much richer than the familiar thermodynamic, Fermi-Dirac or Bose-Einstein spectra and admit in addition finite flux (Kolmogorov-Zakharov) solutions which describe the transfer of conserved densities (e.g. energy) between sources and sinks. There is much one can learn from the kinetic equations about the behavior of particular systems of interest including insights in connection with the phenomenon of intermittency. What we would like to convince you is that what we call weak or wave turbulence is every bit as rich as the macho turbulence of 3D hydrodynamics at high Reynolds numbers and, moreover, is analytically more tractable. It is an excellent paradigm for the study of many-body Hamiltonian systems which are driven far from equilibrium by the presence of external forcing and damping. In almost all cases, it contains within its solutions behavior which invalidates the premises on which the theory is based in some spectral range. We give some new results concerning the dynamic breakdown of the weak turbulence description and discuss the fully nonlinear and intermittent behavior which follows. These results may also be important for proving or disproving the global existence of solutions for the underlying partial differential equations. Wave turbulence is a subject to which many have made important contributions. But no contributions have been more fundamental than those of Volodja Zakharov whose 60th birthday we celebrate at this meeting. He was the first to appreciate that the kinetic equations admit a far richer class of solutions than the fluxless thermodynamic solutions of equilibrium systems and to realize the central roles that finite flux solutions play in non-equilibrium systems. It is appropriate, therefore, that we call these Kolmogorov-Zakharov (KZ) spectra. (C) 2001 Elsevier Science B.V. All rights reserved.
\end{abstract}

Keywords: Wave turbulence; Intermittency; Asymptotic closure; Cumulants

\section{Introduction and motivation}

Turbulence is about understanding the long-time statistical properties of solutions of nonlinear field equations with sources and sinks, and in particular with calculating transport. Sometimes the transport is in physical space such as the flux of heat carried across a layer of fluid by turbulent convection, or the flux of momentum from a fast moving turbulent stream to a fixed plate or the flux of angular momentum across an annulus from an inner rotating cylinder to slower moving outer one. However, the transport can also be in Fourier space, such as the flux of energy from large energy containing scales to small scales where dissipation occurs, and it is the description of this transport that is the main topic of this lecture.

\footnotetext{
${ }^{*}$ Corresponding author.

E-mail address: anewell@maths.warwick.ac.uk (A.C. Newell).

${ }^{1}$ Also at University of Arizona, Tucson, AZ 85721, USA.
} 
The picture we have in mind is this. We have a source of energy at large scales, throughput at middle scales (windows of transparency, inertial ranges) over which the system is essentially conservative and Hamiltonian, and dissipative output at small scales. Among other things, we would like to find solutions of the moment equations for the unforced, undamped system which describe a finite flux of some conserved density, such as energy, across these windows of transparency. This picture derives from the Richardson scenario for the complex vorticity and irregular flow fields encountered in 3D, high Reynolds number hydrodynamics, the granddaddy of turbulent systems. The main role of the energy conserving nonlinear terms (advection and pressure) in the Euler equations is to transfer energy from the large eddies at the integral scale where the system is forced to smaller and smaller eddies and eventually to the viscous sink. If the average kinetic energy $E=\left\langle u^{2}\right\rangle$ is written as $\int_{0}^{\infty} E(k) \mathrm{d} k$, the dissipation rate $P=\left\langle\nu \omega^{2}\right\rangle$ as $2 v \int_{0}^{\infty} k^{2} E(k) \mathrm{d} k$, then the von Karman-Howarth equation is

$$
\frac{\partial E(k, t)}{\partial t}=f(k)+T(k)-2 v k^{2} E(k)
$$

where $f(k), T(k)$ and $2 v k^{2} E(k)$ represent forcing, nonlinear transfer and damping, respectively. $E(k)$ is the angle averaged Fourier transform of the two point velocity correlation function. $T(k)$ is the Fourier transform of third-order moments.

Since the nonlinear transfer $T(k)$ conserves energy, we may write it as the $k$ derivative of a flux, $-\partial P / \partial k$. Then, in the window of transparency,

$$
\frac{\partial}{\partial t} \int_{a}^{b} E \mathrm{~d} k=P_{a}-P_{b}
$$

which can be zero either because $P_{a}=P_{b}=0$ or because $P_{a}=P_{b}=P$, a constant. The first possibility describes an isolated system and leads to an equidistribution of energy in the interval $(a, b)$. The second possibility allows for an energy flux between source and sink, and leads to what is known as the Kolmogorov or finite flux spectrum (see Fig. 1).

However, there is a fundamental difficulty with the moment (cumulant) hierarchy of which (1.1) is the first member. The hierarchy is unclosed and infinite, and no cumulant discard approximations work. Three-dimensional, high Reynolds number hydrodynamics affords the theoretician no useful approximations, no separation of scales, and no footholds for analytical traction. It is too difficult. Simple fluids are easier to drink than they are to understand.

Nevertheless, some progress in understanding turbulent behavior has come as a result of the formidable insights of Kolmogorov. Arguing that the symmetries of translation and isotropy are restored in the statistical sense and that, in the infinite Reynolds number limit, all small-scale statistical properties depend only on the local scale and the energy dissipation rate $P$, one can deduce that

$$
\lim _{r \rightarrow 0} \lim _{\nu \rightarrow 0} \lim _{t \rightarrow \infty}\left\langle\left(u_{\|}(\vec{x}+\vec{r}, t)-u_{\|}(\vec{x}, t)\right)^{n}\right\rangle=C_{n}(\operatorname{Pr})^{n / 3},
$$

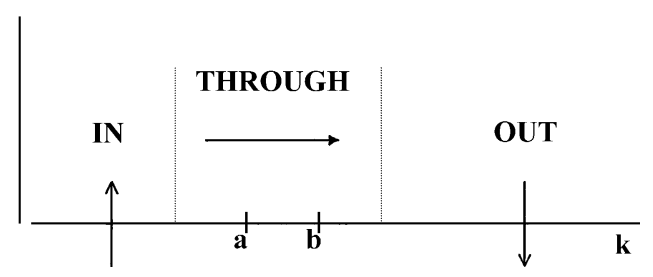

Fig. 1. Forcing, inertial range and damping regions of $k$ space. 
where $u_{\|}=\vec{u} \cdot \hat{r}$ and $C_{n}$ are universal constants. The limits correspond to long time and the necessity of staying away from the sink and source scales, respectively. But, for almost all $n,(1.2)$ is conjecture. From the Navier-Stokes equations, it can only be proved rigorously for $n=3$ [37]. Moreover, recent experimental evidence seems to indicate that the $n$ th-order structure function, $n \geq 4$,

$$
S_{n}(r)=\left\langle(u(\vec{x}+\vec{r})-u(\vec{x}))^{n}\right\rangle
$$

has index less than $\frac{1}{3} n$. If that is the case, the ratio $S_{n} /\left(S_{2}\right)^{n / 2}$, which in the Kolmogorov theory is a pure constant, diverges as $r \rightarrow 0$. The divergence of this ratio indicates that large fluctuations are more probable than Kolmogorov theory would suggest. The present consensus is that fluctuations in the local dissipation rate are responsible for elevating the tails of the probability density function for velocity differences and for what is generally called intermittent behavior.

In contrast, the turbulence of a sea of weakly coupled, dispersive wavetrains has a natural asymptotic closure. One can:

1. find a closed kinetic equation for the spectral energy density;

2. understand the mechanisms (resonance) by which energy and other conserved densities are redistributed throughout the spectrum;

3. obtain stationary solutions of the kinetic equation analogous to both the thermodynamic and Kolmogorov spectra;

4. test the validity of the weak turbulence approximation;

Moreover, the results are not simply a useful paradigm but are of direct interest in their own right in a variety of contexts from optics to oceans, from sound to semiconductor lasers to the solar wind.

The natural closure occurs because of two factors, the weak coupling, $\varepsilon, 0<\varepsilon \ll 1$ and the dispersive nature of the waves. The combination means that there is an effective separation of time scales. On the linear time scale $t_{\mathrm{L}}=\omega_{0}^{-1}, \omega_{0}$ a typical frequency in the initial spectrum, the higher-order cumulants decay towards a state of joint Gaussianity. On the much longer nonlinear time scale $t_{\mathrm{NL}}=\varepsilon^{-(2 r-4)} \omega_{0}^{-1}$, nonlinear resonant interactions of order $r, r=3,4,5$, bring about coherence and a departure from joint Gaussian behavior. The regeneration of the higher-order cumulants by nonlinearity occurs in a special way. For example, in the regeneration of the third-order cumulant (moment), the product of second-order cumulants is more important than the fourth-order cumulant. This pattern continues. The regeneration of cumulants of order $N$ is dominated by products of lower-order ones. This feature leads to the natural closure.

The new message of this paper is to suggest that wave turbulence is an even richer paradigm for non-equilibrium systems than previously believed. The reason is simple and dramatic. As the system relaxes to its asymptotic stationary state, the closure equations almost always become non-uniform at some scale we call $k_{\mathrm{NL}}$. If $k_{\mathrm{NL}}$ lies near the ultraviolet (infrared) end of the spectrum, then for all $k, k>k_{\mathrm{NL}}\left(k<k_{\mathrm{NL}}\right)$, the dynamics become increasingly dominated by large fluctuation local events which are intermittent and fully nonlinear. Some are shock-like; others are spawned by condensate formation. We give explicit formulae for $k_{\mathrm{NL}}$ in terms of properties of the linear dispersion relation, the nonlinear coupling coefficients and the KZ fluxes.

\section{Wave turbulence: asymptotic closure}

The derivation of the closed equations for the long-time behavior of the statistical moments (cumulants) and a discussion of their properties is carried out in a series of seven steps.

1. The set up: the equation for the Fourier amplitudes.

2. Moments, cumulants. 
3. The cumulant (BBGKY) hierarchy.

4. The strategy for solution and the dynamics.

5. Resonant manifolds and asymptotic expansions.

6. The closure equations.

7. Properties: conservation laws, reversibility, finite flux solutions and their temporal formation.

\subsection{The set up: equation for Fourier amplitudes}

Consider a system which in the linear limit admits wavetrain solutions $u^{s}=\exp \left(\vec{i} \vec{k} \cdot \vec{x}+\mathrm{i} \omega^{s}(\vec{k}) t\right)$, where $\omega^{s}(\vec{k})$ is the dispersion relation and $s$ labels the degree of degeneracy which corresponds to the order of the system or the number of frequencies corresponding to a given wavevector $\vec{k}$. Often, $s=+,-$ corresponding to second-order systems where waves travel in one of two directions. By an appropriate choice of canonical variables (sometimes suggested by the Hamiltonian structure), we write

$$
u^{s}(\vec{x}, t)=\int A^{s}(\vec{k}, t) \mathrm{e}^{\mathrm{i} \vec{k} \cdot \vec{x}} \mathrm{~d} \vec{k}, \quad A^{s}(\vec{k}, t)=A_{k}^{s}, \quad A^{s}(\vec{k}, t)=\frac{1}{(2 \pi)^{d}} \int u^{s}(\vec{x}, t) \mathrm{e}^{-\mathrm{i} \vec{k} \cdot \vec{x}} \mathrm{~d} \vec{x},
$$

and find (for $\left.\omega^{s}=s \omega(\vec{k}), s=+,-, \omega(\vec{k})=\omega_{k}\right)$,

$$
\begin{aligned}
\frac{\mathrm{d} A_{k}^{s}}{\mathrm{~d} t}-\mathrm{i} s \omega_{k} A_{k}^{s}= & \varepsilon \sum_{s_{1} s_{2}} \int L_{k k_{1} k_{2}}^{s s_{1} s_{2}} A_{k_{1}}^{s_{1}} A_{k_{2}}^{s_{2}} \delta\left(\vec{k}_{1}+\vec{k}_{2}-\vec{k}\right) \mathrm{d} \vec{k}_{12} \\
& +\varepsilon^{2} \sum_{s_{1} s_{2} s_{3}} \int L_{k_{1} k_{2} k_{3}}^{s s_{1} s_{3}} A_{k_{1}}^{s_{1}} A_{k_{2}}^{s_{2}} A_{k_{3}}^{s_{3}} \delta\left(\vec{k}_{1}+\vec{k}_{2}+\vec{k}_{3}-\vec{k}\right) \mathrm{d} \vec{k}_{123}+\cdots
\end{aligned}
$$

\section{Remark.}

1. Since the class of functions we are dealing with are bounded and non-decaying as $|\vec{x}| \rightarrow \infty, A_{k}^{s}$ is not an ordinary function but a generalized one. However, as we shall see, for spatially homogeneous systems, ensemble averages of products of the Fourier amplitudes have good properties.

2. Notation: $\delta_{12,0}=\delta\left(\vec{k}_{1}+\vec{k}_{2}-\vec{k}\right), \mathrm{d} \vec{k}_{12}=\mathrm{d} \vec{k}_{1} \mathrm{~d} \vec{k}_{2}$, etc.

3. $\varepsilon, 0<\varepsilon \ll 1$, is a measure of nonlinearity.

The system Hamiltonian takes the form

$$
H=\frac{1}{2} \sum_{s} \int \omega_{k} A_{k}^{s} A_{-k}^{-s} \mathrm{~d} \vec{k}+\cdots
$$

\section{Examples.}

Example (a).

1. Optical waves of diffraction in nonlinear media $[32,40,45]$.

2. Superfluids [32,53].

These examples are described by the nonlinear Schrödinger equation for a complex field $u(\vec{x}, t), \vec{x} \in R^{d}, t \in R$.

$$
\begin{aligned}
& \frac{\partial u}{\partial t}+\mathrm{i} \nabla^{2} u+\mathrm{i} a u^{2} u^{*}=0 \quad a \text { constant }, \quad \frac{\partial u}{\partial t}=\mathrm{i} \frac{\delta H}{\delta u^{*}}, \quad H=\int\left(\nabla u \cdot \nabla u^{*}-\frac{a}{2} u^{2} u^{*^{2}}\right) \mathrm{d} \vec{x}, \\
& u(\vec{x}, t)=u^{+}=\int A_{k}^{+} \mathrm{e}^{\mathrm{i} \vec{k} \cdot \vec{x}} \mathrm{~d} \vec{k}, \quad u^{*}(\vec{x}, t)=u^{-}=\int A_{k}^{-} \mathrm{e}^{\mathrm{i} \vec{k} \cdot \vec{x}} \mathrm{~d} \vec{k}, \quad A_{k}^{-}=A_{-k}^{+*} .
\end{aligned}
$$


For fluctuations about the zero state, $u^{s}=0$,

$$
\frac{\partial A_{k}^{s}}{\partial t}-\mathrm{i} s k^{2} A_{k}^{s}=-\mathrm{i} a s \int A_{k_{1}}^{-s} A_{k_{2}}^{s} A_{k_{3}}^{s} \delta_{123,0} \mathrm{~d} \vec{k}_{123} .
$$

Thus, $\varepsilon L_{k k_{1} k_{2}}^{s s_{1} s_{2}}=0, \varepsilon^{2} L_{k k_{1} k_{2} k_{3}}^{s s_{1} s_{2} s_{3}}=-\frac{1}{3} \mathrm{i} a s \mathcal{P}^{123} \delta_{s_{1},-s} \delta_{s_{2}, s} \delta_{s_{3}, s}, \omega_{k}=k^{2}$.

In Fourier coordinates $\left(A_{k}=A_{k}^{+}, A_{k}^{*}=A_{-k}^{-}\right)$

$$
H=\int k^{2} A_{k}^{*} A_{k} \mathrm{~d} \vec{k}-\frac{a}{2} \int A_{k}^{*} A_{k_{1}}^{*} A_{k_{2}} A_{k_{3}} \delta_{01,23} \mathrm{~d} \vec{k}_{0123} .
$$

For fluctuations about the condensate state

$$
u=\rho_{0}^{1 / 2} \mathrm{e}^{-\mathrm{i} a \rho_{0} t}
$$

we rewrite (2.4) in polar coordinates

$$
u=\rho^{1 / 2} \mathrm{e}^{-\mathrm{i} \varphi},
$$

and obtain

$$
\frac{\partial \rho}{\partial t}+2 \nabla \cdot(\rho \nabla \varphi)=0, \quad \frac{\partial \varphi}{\partial t}+|\nabla \varphi|^{2}+(-a) \rho-\frac{1}{\sqrt{\rho}} \nabla^{2}(\sqrt{\rho})=0,
$$

which are the Euler equations for a compressible fluid with velocity field $2 \nabla \varphi$ and pressure $p=-a \rho^{2}$ plus the addition of the extra term $(1 / \sqrt{p}) \nabla^{2}(\sqrt{\rho})$ sometimes called the quantum pressure. Clearly, $\partial p / \partial \rho>0$ only when $a<0$. Take $a=-1$. The Hamiltonian is

$$
H=\int\left(\left(\nabla \rho^{1 / 2}\right)^{2}+\rho(\nabla \varphi)^{2}+\frac{1}{2} \rho^{2}\right) \mathrm{d} \vec{x}
$$

and (2.5) is $\partial \rho / \partial t=\delta H / \delta \varphi, \delta \varphi / \partial t=-\delta H / \delta \rho$. Setting $\rho=\rho_{0}+\delta \rho, \varphi=-\rho_{0} t+\delta \varphi$, one finds to quadratic order that

$$
\frac{\partial}{\partial t} \delta \rho+k_{0}^{2} \nabla^{2} \delta \varphi=-2 \nabla(\delta \rho \nabla \delta \varphi), \quad \frac{\partial}{\partial t} \delta \varphi+\left(1-\frac{1}{k_{0}^{2}} \nabla^{2}\right) \delta \rho=-\frac{1}{k_{0}^{4}} \delta \rho \nabla^{2} \delta \rho-\frac{1}{2 k_{0}^{4}} \nabla^{2}(\delta \rho)^{2}-(\nabla(\delta \varphi))^{2},
$$

where $k_{0}^{2}=2 \rho_{0}$.

Let

$$
\begin{aligned}
& \delta \rho=\int \rho_{k} \mathrm{e}^{\mathrm{i} k \cdot \vec{x}} \mathrm{~d} \vec{k}, \quad \delta \varphi=\int \varphi_{k} \mathrm{e}^{\mathrm{i} \vec{k} \cdot \vec{x}} \mathrm{~d} \vec{k}, \quad \rho_{k}=\left(\frac{k_{0}^{2} k^{2}}{2 \omega_{k}}\right)^{1 / 2} \sum_{s} A_{k}^{s}, \\
& \varphi_{k}=\frac{\mathrm{i}}{2}\left(\frac{2 \omega_{k}}{k_{0}^{2} k^{2}}\right)^{1 / 2} \sum_{s} s A_{k}^{s}, \quad \omega_{k}^{2}=k_{0}^{2} k^{2}+k^{4},
\end{aligned}
$$

and find

$$
\frac{\mathrm{d} A_{k}^{s}}{\mathrm{~d} t}-\mathrm{i} s \omega_{k} A_{k}^{s}=\varepsilon \sum_{s_{1} s_{2}} \int L_{k k_{1} k_{2}}^{s s_{1} s_{2}} A_{k_{1}}^{s_{1}} A_{k_{2}}^{s_{2}} \delta_{12,0} \mathrm{~d} \vec{k}_{12}+\text { cubic terms, }
$$


where

$$
\begin{aligned}
\varepsilon L_{k k_{1} k_{2}}^{s s_{1} s_{2}}= & \frac{\mathrm{i} \sqrt{2}}{4 k_{0}}\left\{s_{1} \vec{k} \cdot \vec{k}_{1}\left(\frac{\omega \omega_{1} k_{2}^{2}}{\omega_{2} k^{2} k_{1}^{2}}\right)^{1 / 2}+s_{2} \vec{k} \cdot \vec{k}_{2}\left(\frac{\omega \omega_{2} k_{1}^{2}}{\omega_{1} k^{2} k_{2}^{2}}\right)^{1 / 2}\right. \\
& \left.+s s_{1} s_{2} \vec{k}_{1} \cdot \vec{k}_{2}\left(\frac{\omega_{1} \omega_{2} k^{2}}{\omega k_{1}^{2} k_{2}^{2}}\right)^{1 / 2}+\left(\frac{k^{2} k_{1}^{2} k_{2}^{2}}{\omega \omega_{1} \omega_{2}}\right)^{1 / 2} s\left(\vec{k}_{1} \cdot \vec{k}_{2}-k^{2}\right)\right\} .
\end{aligned}
$$

Note: $\varepsilon L_{k k_{1}, k_{2}}^{+++}=\mathrm{i} \sqrt{2} V_{k k_{1} k_{2}}$ in Ref. [32]. For $k_{0} \ll k, L \sim L$ because leading terms cancel. For $k_{0} \gg k, L \sim k^{3 / 2}$.

In general, for the class of zero mean conservative systems (2.2) for which $A_{k}^{s}$ and $A_{-k}^{-s}$ are conjugate variables and $A_{k t}^{s}=\mathrm{i} s \delta H / \delta A_{-k}^{-s}$, the following properties hold:

1. $L_{k k_{1} \cdots k_{r}}^{s s_{1} \cdots s_{r}}=-L_{k k_{1} \cdots k_{r}}^{* s s_{1} \cdots s_{r}}=-L_{-k-k_{1} \cdots-k_{r}}^{-s-s_{1} \cdots-s_{r}}$.

2. $L_{k k_{1} \cdots k_{r}}^{s s_{1} \cdots s_{r}}$ is symmetric in $(1,2, \ldots, r)$.

3. $L_{0 k_{1} \cdots k_{r}}^{s s_{1} \cdots s_{r}}=0, \vec{k}_{1}+\cdots+\vec{k}_{r}=0$ (except for NLS).

4. $L_{k_{1} k-k_{2} \cdots-k_{r}}^{s_{1} s-s_{2} \cdots-s_{r}}=\left(s_{1} / s\right) L_{k k_{1} k_{2} \cdots k_{r}}^{s s_{1} s_{2} \cdots s_{r}}$ when $\vec{k}_{1}+\cdots+\vec{k}_{r}=\vec{k}$.

Example (b). Water waves [1,2,4-8,11,12,19,23,34,36,44,48]. For details, see [11] (see Fig. 2).

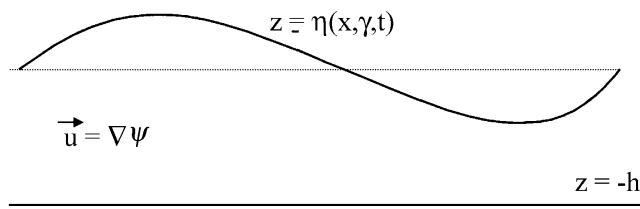

Fig. 2. An ocean of depth $h$, surface deformation $\eta(x, y, t)$ and velocity potential $\psi(x, y, z, t)$.

$$
\begin{aligned}
& \eta(x, y, t)=\eta(\vec{x}, t)=\sum_{s} \int_{-\infty}^{\infty} \sqrt{\frac{\omega_{k}}{2}} \frac{1}{v_{k}} A_{k}^{s} \mathrm{e}^{\mathrm{i} \vec{k} \cdot \vec{x}} \mathrm{~d} \vec{k}, \quad \psi(\vec{x}, t)=\sum_{s} \int_{-\infty}^{\infty} \frac{\mathrm{i} v_{k} s}{\sqrt{2 \omega_{k}}} A_{k}^{s} \frac{\cosh k(z+h)}{\cosh k h} \mathrm{e}^{\mathrm{i} \vec{k} \cdot \vec{x}} \mathrm{~d} \vec{k}, \\
& \omega_{k}=\sqrt{\left(g k+\sigma k^{3}\right) \tanh k h}, v_{k}^{2}=g+\sigma k^{2}, g=\text { gravity, } \sigma=S / \rho=\frac{\text { surface tension }}{\text { density }}, \\
& \langle\text { Energy }\rangle=\sum_{s} \int \omega_{k} Q^{s-s}(k) \mathrm{d} \vec{k}, \quad\left\langle A_{k}^{s} A_{k^{\prime}}^{s^{\prime}}\right\rangle=\delta\left(k+k^{\prime}\right) Q^{s s^{\prime}}\left(k^{\prime}\right) .
\end{aligned}
$$

Also

$$
\begin{aligned}
& \varepsilon L_{k k_{1} k_{2}}^{s s_{1} s_{2}}=\frac{i}{2} \sqrt{\frac{\omega_{1} \omega_{2}}{2 \omega}} \frac{v}{v_{1} v_{2}}\left\{\frac{1}{2} \mathcal{P} \frac{v_{2}^{2}}{s_{2} \omega_{2}}\left(k_{2}^{2}+\vec{k}_{1} \cdot \vec{k}_{2}\right)-\frac{s \omega}{2 v^{2}}\left(\omega_{1}^{2}+\omega_{2}^{2}+s_{1} \omega_{1} s_{2} \omega_{2}-\vec{k}_{1} \cdot \vec{k}_{2} \frac{v_{1}^{2} v_{2}^{2}}{s_{1} \omega_{1} s_{2} \omega_{2}}\right)\right\}, \\
& \varepsilon^{2} L_{k k_{1} k_{2} k_{3}}^{s s_{1} s_{2} s_{3}}=\frac{\mathrm{i}}{2} \sqrt{\frac{\omega_{1} \omega_{2} \omega_{3}}{\omega}} \frac{v}{v_{1} v_{2} v_{3}} \stackrel{123}{\mathcal{P}}\left\{\frac{1}{6} \mathcal{P} s_{3} \omega_{3}\left(\frac{1}{2} k_{3}^{2}+\vec{k}_{2} \cdot \vec{k}_{3}\right)\right. \\
& -\frac{s \omega}{3 v^{2}}\left[\frac{v_{3}^{2} k_{3}^{2}+v_{2}^{2} k_{2}^{2}}{4}+\frac{1}{2} \mathcal{P} \frac{v_{2}^{2} \omega_{3}^{2}\left(k_{2}^{2}-\vec{k}_{2} \cdot \vec{k}_{3}\right)}{s_{2} \omega_{2} s_{3} \omega_{3}}\right]-\sqrt{\frac{\omega_{2} \omega_{3}}{2 \omega}} \frac{v}{v_{2} v_{3}} \frac{s \omega}{6 v^{2}}\left|k_{2}+k_{3}\right| \tanh \mid k_{2} \\
& \left.+k_{3} \mid h\left(\omega_{2}^{2}+\omega_{3}^{2}+s_{2} \omega_{2} s_{3} \omega_{3}-\frac{\vec{k}_{2} \cdot \vec{k}_{3} v_{2}^{2} v_{3}^{2}}{s_{2} \omega_{2} s_{3} \omega_{3}}\right)-\frac{s \omega}{3 v^{2}} \sigma\left(\left(\vec{k}_{1} \times \vec{k}_{2}\right)\left(\vec{k}_{1} \times \vec{k}_{3}\right)-\frac{3}{2} k_{1}^{2} \vec{k}_{2} \cdot \vec{k}_{3}\right)\right\},
\end{aligned}
$$


where $\mathcal{P}^{23}$ is permutation over $(2,3), \mathcal{P}^{123}$ over $(1,2,3)$. For $g \gg \sigma k^{2}, L_{k k_{1} k_{2} k_{3}}^{s s_{1} s_{3}}$ is homogeneous of order 3, i.e. $L_{\varepsilon k}=\varepsilon^{3} L_{k}$. For $g \ll \sigma k^{2}, L_{k k_{1} k_{2}}^{s s_{1} s_{2}}$ is homogeneous of order $\frac{9}{4}$.

Example (c). Sound waves $[13,14,22,38]$.

$$
\begin{aligned}
& p=p_{0}\left(\frac{\rho}{\rho_{0}}\right)^{\mu}, \quad c^{2}=\frac{\mu p_{0}}{\rho_{0}}, \quad \rho=\rho_{0}\left(1+\sum_{s} \int A_{k}^{s} \mathrm{e}^{\mathrm{i} \vec{k} \cdot \vec{x}} \mathrm{~d} \vec{k}\right), \\
& v_{j}=\sum_{s} \int-\frac{c^{2} k_{j}}{s \omega_{k}} A_{k}^{s} \mathrm{e}^{\mathrm{i} \vec{k} \cdot \vec{x}} \mathrm{~d} \vec{k}, \quad \omega_{k}=c k
\end{aligned}
$$

where $p, \rho$ and $v_{j}$ are pressure, density and the $j$ th component of velocity, respectively.

$$
\varepsilon L_{k k_{1} k_{2}}^{s s_{1} s_{2}}=\frac{\mathrm{i} c^{2}}{4}\left(\frac{k \cdot \vec{k}_{1}}{s_{1} \omega_{1}}+\frac{\vec{k} \cdot \vec{k}_{2}}{s_{2} \omega_{2}}+\frac{s \omega}{s_{1} \omega_{1} s_{2} \omega_{2}} \vec{k}_{1} \cdot \vec{k}_{2}\right)+\frac{\mathrm{i}}{4}(\mu-2) s \omega, \quad \varepsilon^{2} L_{k k_{1} k_{2} k_{3}}^{s s_{1} s_{2} s_{3}}=\frac{\mathrm{i} \omega_{k}}{12}(\mu-2)(\mu-3) .
$$

Each is homogeneous in $k$ of degree 1 .

For examples of weak turbulence in semiconductor lasers, magnetohydrodynamics, coupled oscillators, plasmas and atmospheric waves, quantum systems see $[3,9,10,15-18,20,21,24,28,30,31,39,40,45,46,49]$.

\subsection{Moments, cumulants}

But Eq. (2.2) for the Fourier amplitude is only a means to an end. We really want to describe the behavior of moments

$$
M_{N}^{s s^{\prime} \ldots s^{(N-1)}}\left(\vec{x} ; \vec{r}, \vec{r}^{\prime} \vec{r}^{\prime \prime}, \ldots, \vec{r}^{(N-2)} ; t\right)=\left\langle u^{s}(\vec{x}) u^{s^{\prime}}(\vec{x}+\vec{r}) \cdots u^{s^{(N-1)}}\left(\vec{x}+\vec{r}^{(N-2)}\right)\right\rangle
$$

as time $t$ becomes large.

At this stage \langle\rangle denotes an ensemble average connected with a joint probability density function (jpdf) $P\left(u_{0}^{s}, u_{1}^{s^{\prime}}, \ldots, u_{(N-1)}^{s^{(N-1)}}\right)$. Namely, $P\left(u_{0}^{s}, \ldots\right) \mathrm{d} u_{0}^{s} \ldots$ is the probability that the field $u^{s}$ at $x$ lies between $u_{0}^{s}$ and $u_{0}^{s}+\mathrm{d} u_{0}^{s}$, the field $u^{s^{\prime}}$ at $x+r$ lies between $u_{1}^{s^{\prime}}$ and $u_{1}^{s^{\prime}}+\mathrm{d} u_{1}^{s^{\prime}}$, and so on. However, we now make the assumption of spatial homogeneity. $M_{N}$ only depends on the relative geometry of the configuration and does not depend on the base coordinate $\vec{x}$. In this situation, it is convenient to think of \langle\rangle as an average over the base coordinate.

But one should be cautious with the assumption of spatial homogeneity. It is a property of the equations that if $M_{N}$ is independent of the base point $\vec{x}$ initially, it will remain so. But suppose $M_{N}$ depends weakly on $\vec{x}$ initially. Then, the nonlinear dynamics can lead to a steepening of derivatives (cf. Burgers equation) and a possible breakdown of spatial homogeneity into patches separated by shocks. In this essay, we ignore this possibility.

Moments are inconvenient for several reasons. In particular $M_{N}$ does not tend to zero as the separations $\vec{r}, \vec{r}^{\prime}, \ldots$ tend independently to infinity. This means that it does not possess an ordinary Fourier transform which is the coordinate most relevant when dealing with a field of wavetrains. Therefore, we use cumulants which are related in a 1:1 fashion with the moments.

$$
\begin{aligned}
& M_{1}^{s}(t)=\left\langle u^{s}(\vec{x})\right\rangle=R^{(1) s}(t)=\left\{u^{s}(\vec{x})\right\}, \\
& M_{2}^{s s^{\prime}}(\vec{r}, t)=\left\langle u^{s}(\vec{x}) u^{s^{\prime}}(\vec{x}+\vec{r})\right\rangle=\left\{u^{s}(\vec{x}) u^{s^{\prime}}(\vec{x}+\vec{r})\right\}+\left\{u^{s}(\vec{x})\right\}\left\{u^{s^{\prime}}(\vec{x}+\vec{r})\right\}=R^{(2) s s^{\prime}}(\vec{r})+R^{(1) s} R^{(1) s^{\prime}}, \\
& M_{3}^{s s^{\prime} s^{\prime \prime}}\left(\vec{r}, \vec{r}^{\prime}, t\right)=\left\langle u^{s}(\vec{x}) u^{s^{\prime}}(\vec{x}+\vec{r}) u^{s^{\prime \prime}}\left(\vec{x}+\vec{r}^{\prime \prime}\right)\right\rangle=R^{(3) s s^{\prime} s^{\prime \prime}}\left(\vec{r}, \vec{r}^{\prime}\right)+\text { one of all possible partitions, } \\
& M_{N}^{(N) s s^{\prime} \cdots}=R^{(N) s s^{\prime} \cdots}+\text { one of all possible partitions. }
\end{aligned}
$$


Curly brackets denote cumulants, and angle brackets are moments. We will also study systems where $M_{1}=\langle u\rangle \equiv 0$. Then,

$$
\begin{aligned}
& M_{2}^{s s^{\prime}}(\vec{r})=R^{(2) s s^{\prime}}(\vec{r})=\left\langle u^{s}(\vec{x}) u^{s^{\prime}}(\vec{x}+\vec{r})\right\rangle, \\
& M_{3}^{s s^{\prime} s^{\prime \prime}}\left(\vec{r}, \vec{r}^{\prime}\right)=R^{(3) s s^{\prime} s^{\prime \prime}}\left(\vec{r}, \vec{r}^{\prime}\right)=\left\langle u^{s}(\vec{x}) u^{s^{\prime}}(\vec{x}+\vec{r}) u^{s^{\prime \prime}}\left(\vec{x}+r^{\prime}\right)\right\rangle, \\
& M_{4}^{s s^{\prime} s^{\prime \prime} s^{\prime \prime \prime}}\left(\vec{r}, \vec{r}^{\prime}, \vec{r}^{\prime \prime}\right)=R^{(4) s s^{\prime} s^{\prime \prime} s^{\prime \prime \prime}}\left(\vec{r}, \vec{r}^{\prime}, \vec{r}^{\prime \prime}\right)+R^{(2) s s^{\prime}}(\vec{r}) R^{(2) s^{\prime \prime} s^{\prime \prime \prime}}\left(\vec{r}^{\prime \prime}-\vec{r}^{\prime}\right)+R^{(2) s s^{\prime \prime}}\left(\vec{r}^{\prime}\right) R^{(2) s^{\prime} s^{\prime \prime}}\left(\vec{r}^{\prime \prime}-\vec{r}\right)
\end{aligned}
$$

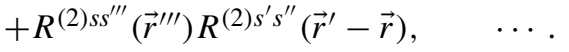

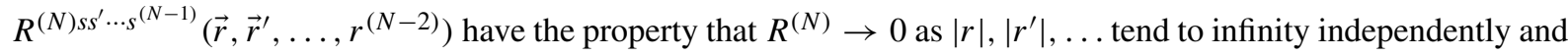
in any directions because it is reasonable to assume, at least at one particular time when the fields are first stirred, that the statistics of widely separated points are uncorrelated.

This means that its Fourier transforms $Q^{(N)}$,

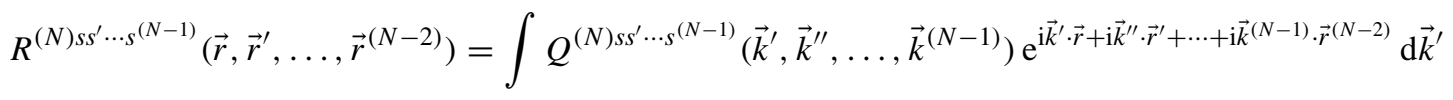

$$
\begin{aligned}
& \times \mathrm{d} \vec{k}^{\prime \prime} \cdots \mathrm{d} \vec{k}^{(N-1)}, \\
& Q^{(N) s s^{\prime} \cdots s^{(N-1)}}\left(\vec{k}^{\prime}, \vec{k}^{\prime \prime}, \ldots, \vec{k}^{(N-1)}\right)=\frac{1}{(2 \pi)^{(N-1) d}} \int R^{(N) s s^{\prime} \cdots s^{(N-1)}}\left(\vec{r}, \vec{r}^{\prime}, \ldots, \vec{r}^{(N-2)}\right) \mathrm{e}^{-\mathrm{i} \vec{k}^{\prime} \cdot \vec{r} \cdots-\mathrm{i} \vec{k}^{(N-1)} \cdot \vec{r}^{(N-2)}} \\
& \times \mathrm{d} \vec{r} \mathrm{~d} \vec{r}^{\prime} \cdots \mathrm{d} \vec{r}^{(N-2)}
\end{aligned}
$$

are smooth functions, at least at one time.

\section{Remark.}

1. The dynamics will produce non-smooth components due to nonlinearities. Indeed, understanding these nonsmooth behaviors is the key to understanding the long-time behavior of wave turbulent fields.

2. The notation of pairing $\vec{k}^{\prime}$ with $\vec{r}, \vec{k}^{\prime \prime}$ with $r^{\prime}, \ldots$ and $\vec{k}^{(N-1)}$ with $\vec{r}^{(N-2)}$ is a matter of future convenience.

\subsection{The cumulant hierarchy}

We now introduce the dynamics by writing down the equations for the Fourier space cumulants $Q^{(2) s s^{\prime}}\left(k^{\prime}\right)$, $Q^{(3) s s^{\prime} s^{\prime \prime}}\left(k^{\prime}, k^{\prime \prime}\right), \ldots, Q^{(N) s s^{\prime} \ldots s^{(N-1)}}\left(k^{\prime}, k^{\prime \prime}, \ldots, k^{(N-1)}\right)$. To do this, we first seek a relationship between averages of products of the Fourier amplitudes $A_{k}^{s}$ and the Fourier cumulants $Q^{(N)}$.

We know already that

$$
A_{k}^{s}=\frac{1}{(2 \pi)^{d}} \int u^{s}(\vec{x}) \mathrm{e}^{-\mathrm{i} \vec{k} \cdot \vec{x}} \mathrm{~d} \vec{x}
$$

is not an ordinary function because $u^{s}(\vec{x})$ does not tend to zero as $|\vec{x}| \rightarrow \infty$. It is simply bounded there.

If $u^{s}(\vec{x})$ were a finite sum of wavetrains, $u^{s}(x)=\sum_{j} A_{j}^{s} \mathrm{e}^{\mathrm{i} \vec{k}_{j} \cdot \vec{x}}$, then $A_{k}^{s}=\sum_{j} A_{j}^{s} \delta\left(\vec{k}-\vec{k}_{j}\right)$, namely the sum of complex numbers times Dirac delta functions. Interacting wavetrains transfer energy via three-wave interactions on a time scale of $\omega_{0} t=\mathrm{O}(1 / \varepsilon)$. To see this, write $A_{k}^{s}$ in the above form, solve (2.2) iteratively and find that the zero denominators first enter when one calculates the first iterate at order $\varepsilon$.

If $u^{s}(\vec{x})$ were to belong to a set of smooth fields which decayed sufficiently rapidly as $|\vec{x}| \rightarrow \infty$, then $A^{s}(\vec{k}, t)$ would be smooth and the asymptotic expansion $A^{s}(\vec{k}, t)=a_{0}^{s}(\vec{k}) \mathrm{e}^{\mathrm{i} s \omega_{k} t}+\varepsilon A_{1}^{s}(\vec{k}, t)+\cdots$ would remain uniformly valid in time. This is because the small denominators appear in integrals in the combination $-\mathrm{i} W_{12,0}^{-1}\left(\exp \left(\mathrm{i} W_{12,0} t\right)-\right.$ 1), $W_{12,0}=s_{1} \omega_{1}+s_{2} \omega_{2}-s \omega$. Even though $W_{12,0}$ can be zero, from Section 2.5 we will see that $A_{1}^{s}(\vec{k}, t)$ is bounded 
in time (we exclude the case of multiple zeros). Thus, if the fields are sufficiently small and decay sufficiently fast as $|\vec{x}| \rightarrow \infty$, for all intents and purposes, (2.2) behaves as a linear system. The physical reason is that resonant wavetrains are not long enough to have enough time to interact to produce order 1 exchanges of energy.

The fields of interest to us here are bounded at infinity and consist of collections of infinitely long wavetrains (or wavepackets). Because they are infinite in extent, they have enough time to exchange energy and produce long-time cumulative effects. However, because they are not collections of discrete wavetrains, there is additional phase mixing and, due to these statistical cancellations, the non-uniformities (coherences) produced by nonlinear interactions take longer to appear and energy is exchanged on the time scale $\varepsilon^{-2} \omega_{0}^{-1}$.

To summarize, $L^{2}$ functions have wavetrains which do not interact long enough to produce order 1 cumulative changes. Discrete wavetrains interact very strongly and, via $r$ wave resonances, exchange order 1 amounts of energy on the time scale $\omega_{0} t=\mathrm{O}\left(\varepsilon^{-r+2}\right)$. Continuous spectra of infinite wavetrains, corresponding to bounded, spatially random fields, have additional mixing which slows the rate of order 1 energy exchange via $r$ wave resonances to the time $\omega_{0} t=\mathrm{O}\left(\varepsilon^{-2(r-2)}\right)$.

Let $u^{s}(\vec{x})$ be a spatially homogeneous random field of zero mean. Then, because $A_{k}^{s}$ is a functional of $u^{s}(\vec{x})$,

$$
\begin{aligned}
& \left\langle A_{k}^{s}\right\rangle=0, \\
& \left\langle A_{k}^{s} A_{k^{\prime}}^{s^{\prime}}\right\rangle=\frac{1}{(2 \pi)^{2 d}} \int\left\langle u^{s}(\vec{x}) u^{s^{\prime}}(\vec{x}+\vec{r})\right\rangle \mathrm{e}^{-\mathrm{i} \vec{k} \cdot \vec{x}-\mathrm{i} \vec{k}^{\prime}(\vec{x}+\vec{r})} \mathrm{d} \vec{x} \mathrm{~d}(\vec{x}+\vec{r}) \\
& \quad=\frac{1}{(2 \pi)^{2 d}} \int R^{(2) s s^{\prime}}(\vec{r}) \mathrm{e}^{-\mathrm{i} \vec{k}^{\prime} \cdot \vec{r}} \mathrm{~d} \vec{r} \int \mathrm{e}^{-\mathrm{i}\left(\vec{k}+\vec{k}^{\prime}\right) \cdot \vec{x}} \mathrm{~d} \vec{x}=\delta\left(\vec{k}+\vec{k}^{\prime}\right) Q^{(2) s s^{\prime}}\left(\vec{k}^{\prime}\right)=\delta\left(\vec{k}+\vec{k}^{\prime}\right) Q^{(2) s s^{\prime}}(-\vec{k}) .
\end{aligned}
$$

Note $R^{(2) s s^{\prime}}(\vec{r})=R^{(2) s^{\prime} s}(-\vec{r})$ because

$$
\left\langle u^{s}(\vec{x}) u^{s^{\prime}}(\vec{x}+\vec{r})\right\rangle=\left\langle u^{s}\left(\vec{x}^{\prime}-\vec{r}\right) u^{s^{\prime}}\left(\vec{x}^{\prime}\right)\right\rangle=R^{s^{\prime} s}(-\vec{r}) .
$$

Likewise

$$
\begin{aligned}
& \left\langle A_{k}^{s} A_{k^{\prime}}^{s^{\prime}} A_{k^{\prime \prime}}^{s^{\prime \prime}}\right\rangle=\frac{\delta\left(\vec{k}+\vec{k}^{\prime}+\vec{k}^{\prime \prime}\right)}{(2 \pi)^{2 d}} \int R^{(3) s s^{\prime} s^{\prime \prime}}\left(\vec{r}, \vec{r}^{\prime}\right) \mathrm{e}^{-\mathrm{i} \vec{k}^{\prime} \cdot \vec{r}-\mathrm{i} \vec{k}^{\prime \prime} \cdot \vec{r}^{\prime}} \mathrm{d} \vec{r} \cdot \mathrm{d} \vec{r}^{\prime}=\delta\left(\vec{k}+\vec{k}^{\prime}+\vec{k}^{\prime \prime}\right) Q^{(3) s s^{\prime} s^{\prime \prime}}\left(\vec{k}^{\prime}, \vec{k}^{\prime \prime}\right),
\end{aligned}
$$

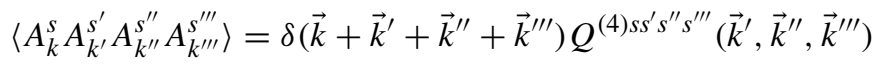

$$
\begin{aligned}
& +\delta\left(\vec{k}+\vec{k}^{\prime}\right) \delta\left(\vec{k}^{\prime \prime}+\vec{k}^{\prime \prime \prime}\right) Q^{(2) s s^{\prime}}\left(\vec{k}^{\prime}\right) Q^{(2) s^{\prime \prime} s^{\prime \prime \prime}}\left(\vec{k}^{\prime \prime \prime}\right) \\
& +\delta\left(\vec{k}+\vec{k}^{\prime \prime}\right) \delta\left(\vec{k}^{\prime}+\vec{k}^{\prime \prime \prime}\right) Q^{(2) s s^{\prime \prime}}\left(\vec{k}^{\prime \prime}\right) Q^{(2) s^{\prime} s^{\prime \prime \prime}\left(\vec{k}^{\prime \prime \prime}\right)} \\
& +\delta\left(\vec{k}+\vec{k}^{\prime \prime \prime}\right) \delta\left(\vec{k}^{\prime}+\vec{k}^{\prime \prime}\right) Q^{(2) s s^{\prime \prime \prime}}\left(\vec{k}^{\prime \prime \prime}\right) Q^{(2) s^{\prime} s^{\prime \prime}}\left(\vec{k}^{\prime \prime}\right)
\end{aligned}
$$

For convenience, we often write

$$
\begin{aligned}
& Q^{(2) s s^{\prime}}\left(\vec{k}^{\prime}\right) \text { as } Q^{(2) s s^{\prime}}\left(\vec{k}, \vec{k}^{\prime}\right) \text {, remembering } \vec{k}+\vec{k}^{\prime}=0 \text { and } \\
& Q^{(3) s s^{\prime} s^{\prime \prime}}\left(\vec{k}^{\prime}, \vec{k}^{\prime \prime}\right) \text { as } Q^{(3) s s^{\prime} s^{\prime \prime}}\left(\vec{k}, \vec{k}^{\prime}, \vec{k}^{\prime \prime}\right) \text {, remembering } \vec{k}+\vec{k}^{\prime}+\vec{k}^{\prime \prime}=0 .
\end{aligned}
$$

This allows one to keep better track of symmetries.

Remark. Note $A_{k}^{s}$ is now not a Dirac delta function as it was when $u^{s}(x)$ was a sum of discrete wavetrains. Its products involve, after averaging, a Dirac delta function times a smooth (at least at some initial time) cumulant. The fact that the cumulants containing products of $\delta$ functions in $\left\langle A_{k}^{s} A_{k^{\prime}}^{s^{\prime}} A_{k^{\prime \prime}}^{s^{\prime \prime}} A_{k^{\prime \prime \prime}}^{s^{\prime \prime \prime}}\right\rangle$ (namely the products of $Q^{(2)}$ 's) have more potency than the fourth-order cumulant makes for a natural asymptotic closure. The regeneration of higher-order cumulants by the dynamics depends only weakly on even higher ones and strongly on products of lower ones. 
We build the cumulant hierarchy by multiplying (2.2) by $A_{k^{\prime}}^{s^{\prime}}$, and (2.2), with $s, k$ replaced by $s^{\prime}, k^{\prime}$, by $A_{k}^{s}$ and adding to find, after averaging

$$
\begin{aligned}
& \frac{\mathrm{d} Q^{(2) s s^{\prime}}\left(\vec{k}^{\prime}\right)}{\mathrm{d} t}-\mathrm{i}\left(s \omega+s^{\prime} \omega^{\prime}\right) Q^{(2) s s^{\prime}}\left(\vec{k}^{\prime}\right) \\
& =\stackrel{00^{\prime}}{\mathcal{P}} \varepsilon \sum_{s_{1} s_{2}} \int L_{k k_{1} k_{2}}^{s s_{1} s_{2}} Q^{(3) s^{\prime} s_{1} s_{2}}\left(\vec{k}_{1}, \vec{k}_{2}\right) \delta_{12,0} \mathrm{~d} \vec{k}_{12}+\stackrel{00^{\prime}}{\mathcal{P}} \varepsilon^{2} \sum_{s_{1} s_{2} s_{3}} \int L_{k k_{1} k_{2} k_{3}}^{s s_{1} s_{3} s_{3}} Q^{(4) s_{1} s_{2} s_{3} s^{\prime}}\left(\vec{k}_{1}, \vec{k}_{2}, \vec{k}_{3}, \vec{k}^{\prime}\right) \delta_{0,123} \mathrm{~d} \vec{k}_{123} \\
& \quad+3 \stackrel{00^{\prime}}{\mathcal{P}} \varepsilon^{2} \sum_{s_{1} s_{2} s_{3}} Q^{(2) s_{3} s^{\prime}}\left(\vec{k}^{\prime}\right) \int L_{k k_{1}-k_{1} k}^{s s_{1} s_{2} s_{3}} Q^{(2) s_{1} s_{2}}\left(-\vec{k}_{1}\right) \mathrm{d} \vec{k}_{1}, \quad \vec{k}+\vec{k}^{\prime}=0 .
\end{aligned}
$$

In (2.9), we have factored out $\delta\left(k+k^{\prime}\right)$ and used the symbol $\mathcal{P}^{00^{\prime}}$ to denote rewriting the following term with $s, k$ replaced by $s^{\prime}, k^{\prime}$ and adding the two contributions together. Multiplying (2.2) by $A_{k^{\prime}}^{s^{\prime}} A_{k^{\prime \prime}}^{s^{\prime \prime}}$ and applying $\mathcal{P}^{00^{\prime} 0^{\prime \prime}}$, we find to order $\varepsilon$

$$
\begin{aligned}
& \frac{\mathrm{d} Q^{(3) s s^{\prime} s^{\prime \prime}}\left(\vec{k}^{\prime}, \vec{k}^{\prime \prime}\right)}{\mathrm{d} t}-\mathrm{i}\left(s \omega+s^{\prime} \omega^{\prime}+s^{\prime \prime} \omega^{\prime \prime}\right) Q^{(3) s s^{\prime} s^{\prime \prime}}\left(\vec{k}^{\prime}, \vec{k}^{\prime \prime}\right) \\
& =\stackrel{00^{\prime} 0^{\prime \prime}}{\mathcal{P}} \varepsilon \sum_{s_{1} s_{2}} \int L_{k k_{1} k_{2}}^{s s_{1} s_{2}} Q^{(4) s^{\prime} s s^{\prime \prime} s_{1} s_{2}}\left(\vec{k}^{\prime \prime}, \vec{k}_{1}, \vec{k}_{2}\right) \delta_{0,12} \mathrm{~d} \vec{k}_{12} \\
& +2 \varepsilon \stackrel{00^{\prime} 0^{\prime \prime}}{\mathcal{P}} \sum_{s_{3} s_{4}} L_{k-k^{\prime}-k^{\prime \prime}}^{s s_{3} s_{4}} Q^{(2) s_{3} s^{\prime}}\left(\vec{k}^{\prime}\right) Q^{(2) s_{4} s^{\prime \prime}}\left(\vec{k}^{\prime \prime}\right)+\mathrm{o}\left(\varepsilon^{2}\right),
\end{aligned}
$$

where $\vec{k}+\vec{k}^{\prime}+\vec{k}^{\prime \prime}=0$.

Note: It is convenient to relabel $s_{1}, s_{2}$ as $s_{3}, s_{4}$ in the second term of the right-hand side of (2.10). In addition, we have used the properties that $L=0$ when $k=0$ and is symmetric over the indices 1,2 .

We continue to build the hierarchy in this way. The reader should write down the equation for $Q^{(4) s s^{\prime} s^{\prime \prime} s^{\prime \prime \prime}}\left(\vec{k}^{\prime}, \vec{k}^{\prime \prime}, \vec{k}^{\prime \prime \prime}\right)$.

Note that the consistency of $\left\langle A_{k}^{s}\right\rangle=0$ requires that $L_{0 k_{1}-k_{1}}^{S s_{1} s_{2}}=0$.

Proof.

$$
\begin{aligned}
& \frac{\mathrm{d}\left\langle A_{k}^{s}\right\rangle}{\mathrm{d} t}-\mathrm{i} s \omega_{k}\left\langle A_{k}^{s}\right\rangle=\varepsilon \sum_{s_{1} s_{2}} \int L_{k k_{1} k_{2}}^{s s_{1} s_{2}}\left\langle A_{k_{1}}^{s_{1}} A_{k_{2}}^{s_{2}}\right\rangle \delta_{0,12} \mathrm{~d} \vec{k}_{12}=\varepsilon \sum_{s_{1} s_{2}} \delta(k) \int L_{0 k_{1} k_{2}}^{s s_{1} s_{2}} Q^{(2) s_{2} s_{1}}\left(k_{1}\right) \mathrm{d} \vec{k}_{1}, \\
& \frac{\mathrm{d}\left\langle A_{k}^{s}\right\rangle}{\mathrm{d} t}=0 \quad \text { when } L_{0 k_{1}-k_{1}}^{s s_{1} s_{2}}=0 .
\end{aligned}
$$

\subsection{The strategy for solutions and the dynamics}

We solve the cumulant hierarchy of which (2.9) and (2.10) are the first two members as an asymptotic expansion in powers of $\varepsilon$, namely we assume

$$
\begin{aligned}
& Q^{(N) s s^{\prime} \cdots s^{(N-1)}}\left(\vec{k}^{\prime}, \ldots, \vec{k}^{(N-1)}\right)=q_{0}^{(N) s s^{\prime} \cdots s^{(N-1)}}\left(\vec{k}^{\prime}, \vec{k}^{\prime \prime}, \ldots, \vec{k}^{(N-1)}, t\right) \exp \left(\mathrm { i } \left(s \omega+s^{\prime} \omega^{\prime}\right.\right. \\
& \left.\left.+\cdots+s^{(N-1)} \omega^{(N-1)}\right) t\right)+\varepsilon Q_{1}^{(N)}+\varepsilon^{2} Q_{2}^{(N)}+\cdots+\varepsilon^{r} Q_{r}^{(N)}+\mathrm{o}\left(\varepsilon^{r}\right) .
\end{aligned}
$$

This means that the difference between $Q^{(N)}$ and any finite sum, say up to $\varepsilon^{r}$, divided by $\varepsilon^{r}$, tends to zero as $\varepsilon \rightarrow 0$. We will insist that (2.11) is a valid asymptotic expansion for all time $t$. To achieve this, we will have to allow $q_{0}^{(N)}$ to vary slowly in time. 


\section{Remark.}

1. Because of non-smoothness which will appear in $Q_{1}^{(3)}, Q_{2}^{(4)}$ in the long-time limit, it is better to interpret the statement of asymptotic expansions in physical space. Namely, we order $Q^{(N)}$ where it makes sense and, where it does not, we order the corresponding $R^{(N)}$.

2. We are seeking asymptotic and not convergent series representations of the solutions to (2.9) and (2.10)!

We recognize that, because of small denominators which will occur on resonant manifolds $M$ defined by

$$
\vec{k}_{1}+\vec{k}_{2}=\vec{k}, \quad s_{1} \omega\left(\vec{k}_{1}\right)+s_{2} \omega\left(\vec{k}_{2}\right)=s \omega(\vec{k}),
$$

non-uniformities in the asymptotic expansions will arise. For example, we will find

$$
\lim _{\substack{t \rightarrow \infty \\ \varepsilon^{2} t \text { fixed }}} Q_{2}^{(2) s s^{\prime}}\left(\vec{k}^{\prime}\right)=t \tilde{Q}_{2}^{(2) s s^{\prime}}\left(\vec{k}^{\prime}\right)+\hat{Q}_{2}^{(2) s s^{\prime}}\left(\vec{k}^{\prime}\right)
$$

will contain terms which grow with $t$ and terms which are bounded. As a result, for times $\varepsilon^{2} t=\mathrm{O}(1)$, the asymptotic expansion is not well ordered. We restore the well-ordered asymptotic expansion by recognizing that, because of these secular terms, there will be an order 1 transfer in spectral energy (i.e. in $Q^{(2) s s^{\prime}}\left(\vec{k}^{\prime}\right), s^{\prime}=-s$ ) over long times.

This transfer is captured by seeking an asymptotic expansion for the time derivative of $q_{0}^{(N) s s^{\prime} \ldots s^{(N-1)}}\left(\vec{k}^{\prime}, \ldots\right.$, $\left.\vec{k}^{(N-1)}\right)$ as

$$
\begin{aligned}
& \frac{\mathrm{d} q_{0}^{(N) s s^{\prime} \cdots s^{(N-1)}}\left(\vec{k}^{\prime}, \ldots, \vec{k}^{(N-1)}\right)}{\mathrm{d} t}=\varepsilon F_{1}^{(N) s s^{\prime} \cdots s^{(N-1)}}\left(\vec{k}^{\prime}, \ldots, \vec{k}^{(N-1)}\right) \\
& +\varepsilon^{2} F_{2}^{(N) s s^{\prime} \cdots s^{(N-1)}}\left(k^{\prime}, \ldots, k^{(N-1)}\right)+\cdots,
\end{aligned}
$$

and choosing $F_{1}^{(N)}, F_{2}^{(N)}, \ldots$ so as to remove secular terms in (2.11) and render it a well-ordered asymptotic expansion uniform in time $t$. Eq. (2.13) is the equation of asymptotic closure. This is closed because

- $N=2, s^{\prime}=-s, F_{1}^{(2)}=0, F_{2}^{(2)}$ depends only on $q_{0}^{-s s}(k)=n_{k}(t)$. This is the kinetic equation and we write it as

$$
\frac{\mathrm{d} n_{k}}{\mathrm{~d} t}=T_{2}\left[n_{k}\right]+T_{4}\left[n_{k}\right]+\cdots .
$$

- $N=2, s^{\prime}=s$ and $N>2, F_{1}^{(N)}=0, F_{2}^{(N)}=\mathrm{i} q_{0}^{(N) s s^{\prime} \cdots s^{(N-1)}}\left(\vec{k}^{\prime}, \ldots, \vec{k}^{(N-1)}\right)\left(\Omega_{k}^{s}+\cdots+\Omega_{k^{(N-1)}}^{s^{(N-1)}}\right)$ which is solved by frequency renormalization

$$
s \omega_{k} \rightarrow s \omega_{k}+\varepsilon^{2} \Omega_{k}^{s}+\cdots .
$$

We, therefore, achieve a natural asymptotic closure for the cumulant hierarchy.

It is natural because it does not assume anything about the initial statistical distributions of the fields. They need not be joint Gaussian. What happens is that the linear behavior of the system brings the system close to a state of joint Gaussianity. Non-joint Gaussianity is regenerated by nonlinear interactions, e.g. d $Q^{(3)} / \mathrm{d} t$ depends on $Q^{(4)}$ and products of $Q^{(2)}$. However, it is the products of the lower-order cumulants that give a cumulative long-time effect and therefore only they play a role in the long-time dynamics. Hence, one achieves closure.

It is asymptotic because the closure equations (2.13)-(2.15) are long-time "average" equations. They are solved iteratively. To find the behavior for times $\omega_{0} t=\mathrm{o}\left(\varepsilon^{-2}\right)$ one solves the truncated equation (2.14) with $T_{4}, T_{6}$ ignored. To find the behavior for $\omega_{0} t=\mathrm{o}\left(\varepsilon^{-4}\right)$ one solves (2.14) with $T_{6}, T_{8}, \ldots$ ignored, and so on. 
It is important to make several points.

1. Although $T_{2 N}\left[n_{k}\right], N \geq 1$, is formally of order $\varepsilon^{2 N}$ in (2.14) and $\varepsilon^{2 N} \Omega_{2 N k}^{s}, N \geq 1$, is formally of order $\varepsilon^{2 N}$ in (2.15), the terms in the series are still functions of $k$. It turns out that the ratios $T_{2 N+2} / T_{2 N}, \varepsilon^{2} \Omega_{2 N+2 k}^{s} / \Omega_{2 N k}^{s}$, formally of order $\varepsilon^{2}$, do not in general remain uniformly bounded as $k \rightarrow 0$ or $k \rightarrow \infty$ when calculated on solutions of the truncated equations. This fact is the origin of the breakdown of wave turbulence at small and large scales. We return to this in Section 4.

2. The term $T_{2}$ is the kinetic equation (2.14) contains triad (three-wave) resonances. The term $T_{4}$ has been calculated [12] and contains four-wave resonances and gradients (with respect to $\vec{k}$ ) of three-wave resonances and principal part integrals. It is not clear how many of these terms can be reabsorbed into $T_{2}$ by renormalizing the frequency in the exponent of the Dirac delta function. Often the successive terms in the series are schematically represented in diagram notation.

3. The imaginary part of the renormalized series (2.15) for $s \omega_{k}$ has positive imaginary part at o( $\left.\varepsilon^{2}\right)$ if there are three-wave resonances, at o $\left(\varepsilon^{4}\right)$ if there are four-wave resonances, and so on. This means that, over long times, and due to resonant interactions, the zeroth-order Fourier space cumulants (which essentially correspond to initial data), $N=2, s^{\prime}=s, N \geq 3$, decay exponentially. Their physical space counterparts decay for two reasons. They decay algebraically in $t$ because of the Riemann-Lebesgue lemma and the presence of fast oscillations multiplying the zeroth-order Fourier space cumulants.

These remarks must be revisited in those regions of $\vec{k}$ space where the weak turbulence closure (2.14) and (2.15) is non-uniform in $k=|\vec{k}|$.

\subsection{Resonant manifolds and asymptotic expansions}

Solve (2.9) and (2.10), . . iteratively.

$$
\varepsilon^{0}:\left\{\begin{array}{l}
Q_{0}^{(2) s s^{\prime}}\left(\vec{k}^{\prime}, t\right)=q_{0}^{(2) s s^{\prime}}\left(\vec{k}^{\prime}, t\right) \mathrm{e}^{\mathrm{i}\left(s \omega+s^{\prime} \omega^{\prime}\right) t}, \quad \vec{k}+\vec{k}^{\prime}=0 \\
Q_{0}^{(3) s s^{\prime} s^{\prime \prime}}\left(\vec{k}^{\prime}, \vec{k}^{\prime \prime}, t\right)=q_{0}^{(3) s s^{\prime} s^{\prime \prime}}\left(\vec{k}^{\prime}, \vec{k}^{\prime \prime}, t\right) \mathrm{e}^{\mathrm{i}\left(s \omega+s^{\prime} \omega^{\prime}+s^{\prime \prime} \omega^{\prime \prime}\right) t}, \quad \vec{k}+\vec{k}^{\prime}+\vec{k}^{\prime \prime}=0 \\
\vdots \\
Q_{0}^{(N) s s^{\prime} \cdots s^{(N-1)}}\left(\vec{k}^{\prime}, \vec{k}^{\prime \prime}, \ldots, \vec{k}^{(N-1)}\right)=q_{0}^{(N) s s^{\prime} \cdots s^{(N-1)}}\left(\vec{k}^{\prime} \ldots \vec{k}^{(N-1)}, t\right) \mathrm{e}^{\mathrm{i}\left(s \omega+\cdots+s^{(N-1)} \omega^{(N-1)}\right) t} \\
\vec{k}+\cdots+\vec{k}^{(N-1)}=0 .
\end{array}\right.
$$

Note:

- For $N=2, s^{\prime}=-s$, the oscillatory dependence disappears because $\omega(-\vec{k})=\omega^{\prime}=\omega \cdot q_{0}^{(2) s-s}\left(\vec{k}^{\prime}, t\right)$ is proportional to the spectral energy density.

- In anticipation of non-uniformities in (2.11), we allow the coefficients $q_{0}^{(N)}$ vary slowly in time via (2.13).

$$
\varepsilon: \quad Q_{1}^{(2) s s^{\prime}}\left(k^{\prime}, t\right)=\stackrel{00^{\prime}}{\mathcal{P}} \sum_{s_{1} s_{2}} \int L_{k k_{1}, k_{2}}^{s s_{1} s_{2}} q_{0}^{(3) s^{\prime} s_{1} s_{2}}\left(\vec{k}_{1}, \vec{k}_{2}\right) \Delta\left(W_{12,0}\right) \mathrm{e}^{\mathrm{i}\left(s \omega+s^{\prime} \omega^{\prime}\right) t} \delta_{0,12} \mathrm{~d} \vec{k}_{12},
$$

where $\Delta(x)=\left(\mathrm{e}^{\mathrm{i} x t}-1\right) / \mathrm{i} x, W_{12,0}=s_{1} \omega_{1}+s_{2} \omega_{2}-s \omega$. We need to consider long-time behavior of integrals of the form

$$
\int F\left(\vec{k}_{1}\right) \frac{\mathrm{e}^{\mathrm{i} h\left(\vec{k}_{1} ; k\right) t}-1}{\mathrm{i} h\left(\vec{k}_{1} ; \vec{k}\right)} \mathrm{d} \vec{k}_{1}
$$


where $h\left(\vec{k}_{1} ; \vec{k}\right)=s_{1} \omega\left(\vec{k}_{1}\right)+s_{2} \omega\left(\vec{k}-\vec{k}_{1}\right)-s \omega(\vec{k})$. The integrals are dominated by values of $\vec{k}_{1}$ near a zero of $h\left(\vec{k}_{1} ; \vec{k}\right)$, the resonant manifold $M$ defined by $(2.12)$,

$$
\vec{k}_{1}+\vec{k}_{2}=\vec{k}, \quad s_{1} \omega\left(\vec{k}_{1}\right)+s_{2} \omega\left(\vec{k}_{2}\right)=s \omega(\vec{k}) \quad \text { for some } s_{1}, s_{2}, s .
$$

In the neighborhood of

$$
M: \quad h\left(\vec{k}_{1} ; \vec{k}\right)=0,
$$

we coordinatize $\vec{k}_{1}$ (say $k_{1 x}, k_{1 y}$ if $\vec{k}_{1}$ is $2 \mathrm{D}$ in a basis locally parallel and perpendicular to $M$. Let $\vec{k}_{1}^{(0)} \in M$. Then

$$
h\left(\vec{k}_{1} ; \vec{k}\right)=h\left(\vec{k}_{1}^{(0)} ; \vec{k}\right)+\left(\vec{k}_{1}-\vec{k}_{1}^{(0)}\right) \cdot \nabla_{\vec{k}_{1}} h\left(\vec{k}_{1}^{(0)} ; \vec{k}\right)+\cdots
$$

In what follows, we will assume that on $M$,

$$
\nabla_{\vec{k}_{1}} h\left(\vec{k}_{1}^{(0)} ; \vec{k}\right) \neq 0 \quad \text { for } \vec{k}_{1}^{(0)} \in M,
$$

and illustrate by example what happens when $\nabla_{\vec{k}_{1}} h=0$. Calling the local perpendicular coordinate by $x$, the above integral can be written as

$$
\int \mathrm{d} y \int f(y, x) \frac{\mathrm{e}^{\mathrm{i} x t}-1}{\mathrm{i} x} \mathrm{~d} x .
$$

Therefore, we study

$$
\lim _{t \rightarrow \infty} \int f(x) \frac{\mathrm{e}^{\mathrm{i} x t}-1}{\mathrm{i} x} \mathrm{~d} x
$$

In $Q_{1}^{(2)}$, the function $f(x)$ (proportional to $q_{0}^{(3) s^{\prime} s_{1} s_{2}}\left(\vec{k}_{1}, \vec{k}_{2}\right)$ ) is slowly varying in time so the limit is taken in the sense that $t \rightarrow \infty, \varepsilon^{r} t$ fixed for some $r=1,2, \ldots$ The smoothness of $q_{0}^{(3) s^{\prime} s_{1} s_{2}}\left(\vec{k}_{1}, \vec{k}_{2}\right)$ is also important. At the initial time, we have assumed this. If we are to recalculate beginning at some later time $t_{1}=\mathrm{O}\left(1 / \varepsilon^{2}\right)$, an $\mathrm{O}(\varepsilon)$ non-smoothness will have developed in the new "initial" state. We will discuss this and show that for $\lim _{t-t_{1} \rightarrow+\infty}$ it does not contribute whereas for $\lim _{t-t_{1} \rightarrow-\infty}$ it does. This is important in resolving an apparent irreversibility paradox. A little calculation (which can be done many ways, e.g. let $f(x)=f(x)-f(0) \mathrm{e}^{-x^{2}}+f(0) \mathrm{e}^{-x^{2}}$ ) reveals

$$
\lim _{|t| \rightarrow \infty} \int f(x) \frac{\mathrm{e}^{\mathrm{i} x t}-1}{\mathrm{i} x} \mathrm{~d} x=\pi \operatorname{sgnt} f(0)+\mathrm{i} P \int \frac{f(x)}{x} \mathrm{~d} x,
$$

where

$$
\operatorname{sgnt}= \begin{cases}+1, & t>0, \\ -1, & t<0,\end{cases}
$$

and $P$ denotes the Cauchy principal value. Schematically

$$
\lim _{|t| \rightarrow \infty} \Delta(x)=\pi \operatorname{sgnt} \delta(x)+\mathrm{i} P\left(\frac{1}{x}\right) .
$$

Returning to $Q_{1}^{(2) s s^{\prime}}\left(\vec{k}^{\prime}, t\right)$, we see that it is bounded as $t \rightarrow \infty$. This is important. A non-zero $q_{0}^{(3) s^{\prime} s_{1} s_{2}}\left(\vec{k}_{1}, \vec{k}_{2}\right)$ at the initial time (here taken to be $t=0$ ) does not affect the uniformity of (2.11). We can begin with initial conditions far from joint Gaussian. This was first pointed out by Benney and Saffman [4]. 
Next, we calculate

$$
\begin{aligned}
Q_{1}^{(3) s s^{\prime} s^{\prime \prime}}\left(\vec{k}^{\prime}, \vec{k}^{\prime \prime}\right)= & \stackrel{00^{\prime} 0^{\prime \prime}}{\mathcal{P}} \sum_{s_{1} s_{2}} \int L_{k k_{1} k_{2}}^{s s_{1} s_{2}} q_{0}^{(4) s^{\prime} s^{\prime \prime} s_{1} s_{2}}\left(\vec{k}^{\prime \prime}, \vec{k}_{1}, \vec{k}_{2}\right) \Delta\left(W_{12,0}\right) \mathrm{e}^{\mathrm{i}\left(s \omega+s^{\prime} \omega^{\prime}+s^{\prime \prime} \omega^{\prime \prime}\right) t} \delta_{0,12} \mathrm{~d} \vec{k}_{12} \\
& +2 \sum_{s_{3} s_{4}} L_{k-k^{\prime}-k^{\prime \prime}}^{s s_{3} s_{4}} q_{0}^{(2) s_{3} s^{\prime}}\left(\vec{k}^{\prime}\right) q_{0}^{(2) s_{4} s^{\prime \prime}}\left(\vec{k}^{\prime \prime}\right) \Delta\left(s_{3} \omega^{\prime}+s_{4} \omega^{\prime \prime}-s \omega\right) \mathrm{e}^{\mathrm{i}\left(s \omega+s^{\prime} \omega^{\prime}+s^{\prime \prime} \omega^{\prime \prime}\right) t} \\
& +2 \sum_{s_{3} s_{4}} L_{k^{\prime}-k^{\prime \prime}-k}^{s^{\prime} s_{3} s_{4}} q_{0}^{(2) s_{3} s^{\prime \prime}}\left(\vec{k}^{\prime \prime}\right) q_{0}^{(2) s_{4} s}(\vec{k}) \Delta\left(s_{3} \omega^{\prime \prime}+s_{4} \omega-s^{\prime} \omega^{\prime}\right) \mathrm{e}^{\mathrm{i}\left(s \omega+s^{\prime} \omega^{\prime}+s^{\prime \prime} \omega^{\prime \prime}\right) t} \\
& +2 \sum_{s_{3} s_{4}} L_{k^{\prime \prime}-k-k^{\prime}}^{s^{\prime \prime} s_{3} s_{4}} q^{(2) s_{3} s}(\vec{k}) q_{0}^{(2) s_{4} s^{\prime}}\left(\vec{k}^{\prime}\right) \Delta\left(s_{3} \omega+s_{4} \omega^{\prime}-s^{\prime \prime} \omega^{\prime \prime}\right) \mathrm{e}^{\mathrm{i}\left(s \omega+s^{\prime} \omega^{\prime}+s^{\prime \prime} \omega^{\prime \prime}\right) t}
\end{aligned}
$$

Note: Because there are no integrals left on some of these terms, and because they contain oscillating factors, their long-time limit cannot be directly taken. We, therefore, return to physical space and examine instead the asymptotic well orderedness of the corresponding physical space cumulant $R^{(3)}$. Schematically, however, we can write $\lim _{t \rightarrow \infty} \Delta(x)=\pi \operatorname{sgnt} \delta(x)+\mathrm{i} P(1 / x)$. The integral in physical space will generally be the product of bounded and oscillatory factors which tend to zero because of the Riemann-Lebesgue lemma. However, for $s_{3}=-s^{\prime}, s_{4}=$ $-s^{\prime \prime}$ in the first, $s_{3}=-s^{\prime \prime}, s_{4}=-s$ in the second, and $s_{3}=-s, s_{4}=-s^{\prime}$ in the third, the exponents coalesce $\Delta\left(-s \omega-s^{\prime} \omega^{\prime}-s^{\prime \prime} \omega^{\prime \prime}\right) \mathrm{e}^{\mathrm{i}\left(s \omega+s^{\prime} \omega^{\prime}+s^{\prime \prime} \omega^{\prime \prime}\right) t}=\Delta\left(s \omega+s^{\prime} \omega^{\prime}+s^{\prime \prime} \omega^{\prime \prime}\right)$ and these terms survive the $t \rightarrow \infty$ limit.

In Section 4, we will calculate these surviving terms for each $R^{(N)}$ and examine their asymptotic expansions for well orderedness. We will find that, consistent with Remark 1 in Section 2.4, they are not always well ordered. Non-uniformities in the corresponding asymptotic expansions for the structure functions can appear at both small and large separations. This can lead to intermittent behavior dominated by fully nonlinear solutions of the field equations. We will discuss this further in Section 4.

As $t \rightarrow \pm \infty$,

$$
\begin{aligned}
Q_{1}^{(3) s s^{\prime} s^{\prime \prime}}\left(\vec{k}^{\prime}, \vec{k}^{\prime \prime}\right) \rightarrow \tilde{Q}_{1}^{(3) s s^{\prime} s^{\prime \prime}}\left(\vec{k}^{\prime}, \vec{k}^{\prime \prime}\right)= & 2\left(\pi \operatorname{sgnt} \delta\left(s \omega+s^{\prime} \omega^{\prime}+s^{\prime \prime} \omega^{\prime \prime}\right)+\mathrm{i} P\left(\frac{1}{s \omega+s^{\prime} \omega^{\prime}+s^{\prime \prime} \omega^{\prime \prime}}\right)\right) \\
& \left.\cdot\left(\stackrel{00^{\prime} 0^{\prime \prime}}{\mathcal{P}} L_{k-k^{\prime}-k^{\prime \prime}}^{s-s^{\prime}-q_{0}^{\prime \prime}}\right)(2)-s^{\prime} s^{\prime}\left(\vec{k}^{\prime}\right) q_{0}^{(2)-s^{\prime \prime} s^{\prime \prime}}\left(\vec{k}^{\prime \prime}\right)\right) .
\end{aligned}
$$

In our calculation of $Q_{2}^{(2) s s^{\prime}}\left(\vec{k}^{\prime}\right)$, however, we will find that it is $Q_{1}^{(3) s^{\prime} s_{1} s_{2}}\left(\vec{k}_{1}, \vec{k}_{2}\right) \mathrm{e}^{-\mathrm{i}\left(s \omega+s^{\prime} \omega^{\prime}\right) t}$ which appears in the integrand and it is its long-time limit that we will need.

Remark. We now, at $\mathrm{O}(\varepsilon)$, have seen the appearance of irreversibility in a reversible system. The limit process effectively ignores all the fluctuating terms so that information on phase is effectively lost in taking the limit. Solutions of the resulting kinetic equations can be attractors.

Before we complete the calculations by identifying the secular terms which appear at $\mathrm{O}\left(\varepsilon^{2}\right)$ and thereby calculate the kinetic equation and renormalization factors (i.e. $F_{2}^{(N)}$ for $N \geq 2$ ), let us return to the study of (2.15) and illustrate in Example 2 what happens if $\nabla h \cdot \hat{n}=0(\hat{n}$ unit normal to $M)$.

Example $1\left(\omega=k^{2}\right)$. Take $\vec{k}=\left(k_{1}, 0\right) ; \vec{k}_{1}=\left(k_{1 x}, k_{1 y}\right)$,

$$
M: \quad h=k_{1}^{2}+\left(\vec{k}-\vec{k}_{1}\right)^{2}-k^{2}=2\left(\left(k_{1 x}-\frac{1}{2} k\right)^{2}+k_{1 y}^{2}-\frac{1}{4} k^{2}\right) .
$$

The resonant manifold for $\vec{k}$ is $M(\vec{k})$ and it is the set of $\vec{k}_{1}$ for which $h=0$. It is a circle of radius $\frac{1}{2} k$ centered at the middle of the vector $\vec{k}$. We can coordinatize $M$ as follows: $k_{1 x}=k \cos ^{2}(\theta / 2), k_{1 y}=k \sin (\theta / 2) \cos (\theta / 2), \nabla h=$ $2\left(k_{1 x}-\frac{1}{2} k\right), 2 k_{1 y}=k(\cos \theta, \sin \theta)$ is never identically zero $(\nabla h \cdot \hat{t}=0, \nabla h \cdot \hat{n} \neq 0)$. 
Example 2 (Acoustic waves: $\omega=|\vec{k}|$ ). Here $h=s_{1}\left|\vec{k}_{1}\right|+s_{2}\left|\vec{k}-\vec{k}_{1}\right|-s|\vec{k}|$ is zero when $\vec{k}_{1}$ is collinear with $\vec{k}$. Now $\nabla h=0$ for all $\vec{k}_{1} \in M$. The integral (2.15) now depends critically on dimension. In $2 \mathrm{D}, \int f(x)\left(\left(\mathrm{e}^{\mathrm{i} h t}-1\right) / \mathrm{i} h\right) \mathrm{d} \vec{k}_{1} \sim$ $\int f(x)\left(\left(\mathrm{e}^{\mathrm{i} x^{2} t}-1\right) / \mathrm{i} x^{2}\right) \mathrm{d} x \sim t^{1 / 2}$. In 3D, $\int f(x, y)\left(\mathrm{e}^{\mathrm{i}\left(x^{2}+y^{2}\right) t} / \mathrm{i}\left(x^{2}+y^{2}\right)\right) \mathrm{d} x \mathrm{~d} y \sim \ln t$ (see [13,14,38]).

Remark. The web created by the manifolds $M(\vec{k}), M\left(\vec{k}_{1}\right), M\left(\vec{k}_{2}\right), \ldots$, where $\vec{k}_{1} \in M(\vec{k}), \vec{k}_{2} \in M\left(\vec{k}_{1}\right), \ldots$, has an interesting geometry [42].

\subsection{The closure equations}

We now come to the final step in the closure calculation, namely the identification of the first terms in (2.11) which are non-uniform, the choices for $F_{1}^{(N)}, F_{2}^{(N)}$ and the resulting equations for asymptotic closure.

The equation for $Q_{2}^{(2) s s^{\prime}}\left(\vec{k}^{\prime}\right)$ is

$$
\frac{\mathrm{d}}{\mathrm{d} t}\left(Q_{2}^{(2) s s^{\prime}}\left(\vec{k}^{\prime}\right) \mathrm{e}^{-\mathrm{i}\left(s \omega+s^{\prime} \omega^{\prime}\right) t}\right)+F_{2}^{(2) s s^{\prime}}\left(\vec{k}^{\prime}\right)=\stackrel{00^{\prime}}{\mathcal{P}} \sum_{s_{1} s_{2}} \int L_{k k_{1} k_{2}}^{s s_{1} s_{2}}\left(Q_{1}^{(3) s^{\prime} s_{1} s_{2}}\left(\vec{k}_{1}, \vec{k}_{2}\right) \mathrm{e}^{-\mathrm{i}\left(s \omega+s^{\prime} \omega^{\prime}\right) t}\right) \delta_{0,12} \mathrm{~d} \vec{k}_{12}
$$

From (2.16) (the appearance of the indices 1, 2 here necessitated the use of the dummy indices 3, 4 in (2.16)),

$$
\begin{aligned}
& Q_{1}^{(3) s^{\prime} s_{1} s_{2}}\left(\vec{k}^{\prime}, \vec{k}_{1}, \vec{k}_{2}\right) \mathrm{e}^{-\mathrm{i}\left(s \omega+s^{\prime} \omega^{\prime}\right) t} \\
& =\stackrel{1}{\mathcal{P}}^{120^{\prime}} \sum_{s_{3} s_{4}} \int L_{k^{\prime} k_{3} k_{4}}^{s^{\prime} s_{3} s_{4}} q_{0}^{(4) s_{1} s_{2} s_{3} s_{4}}\left(\vec{k}_{2}, \vec{k}_{3}, \vec{k}_{4}\right) \Delta\left(W_{34,0^{\prime}}\right) \mathrm{e}^{\mathrm{i}\left(s^{\prime} \omega^{\prime}+s_{1} \omega_{1}+s_{2} \omega_{2}\right) t} \delta_{0^{\prime}, 34} \mathrm{~d} \vec{k}_{34} \cdot \mathrm{e}^{-\mathrm{i}\left(s \omega+s^{\prime} \omega^{\prime}\right) t} \\
& \quad+2 \sum_{s_{3} s_{4}} L_{k^{\prime}-k_{1}-k_{2}}^{s^{\prime} s_{3} s_{4}} q_{0}^{(2) s_{3} s_{1}}\left(\vec{k}_{1}\right) q_{0}^{(2) s_{4} s_{2}}\left(\vec{k}_{2}\right) \Delta\left(s_{3} \omega_{1}+s_{4} \omega_{2}-s^{\prime} \omega^{\prime}\right) \mathrm{e}^{\mathrm{i}\left(s^{\prime} \omega^{\prime}+s_{1} \omega_{1}+s_{2} \omega_{2}\right) t} \mathrm{e}^{-\mathrm{i}\left(s \omega+s^{\prime} \omega^{\prime}\right) t} \\
& \quad+2 \sum_{s_{3} s_{4}} L_{k_{1}-k_{2}-k^{\prime}}^{s_{1} s_{3} s_{4}} q_{0}^{(2) s_{3} s_{2}}\left(\vec{k}_{2}\right) q_{0}^{(2) s_{4} s^{\prime}}\left(\vec{k}^{\prime}\right) \Delta\left(s_{3} \omega_{2}+s_{4} \omega^{\prime}-s_{1} \omega_{1}\right) \mathrm{e}^{\mathrm{i}\left(s^{\prime} \omega^{\prime}+s_{1} \omega_{1}+s_{2} \omega_{2}\right) t} \mathrm{e}^{-\mathrm{i}\left(s \omega+s^{\prime} \omega^{\prime}\right) t} \\
& \quad+2 \sum_{s_{3} s_{4}} L_{k_{2}-k^{\prime}-k_{1}}^{s_{2} s_{3} s_{4}} q_{0}^{(2) s_{3} s^{\prime}}\left(\vec{k}^{\prime}\right) q_{0}^{(2) s_{4} s_{1}}\left(\vec{k}_{1}\right) \Delta\left(s_{3} \omega^{\prime}+s_{4} \omega_{1}-s_{2} \omega_{2}\right) \mathrm{e}^{\mathrm{i}\left(s^{\prime} \omega^{\prime}+s_{1} \omega_{1}+s_{2} \omega_{2}\right) t} \mathrm{e}^{-\mathrm{i}\left(s \omega+s^{\prime} \omega^{\prime}\right) t} .
\end{aligned}
$$

Note: $\delta\left(\vec{k}^{\prime}-\vec{k}_{3}-\vec{k}_{4}\right)$ combined with $\delta\left(\vec{k}_{1}+\vec{k}_{2}+\vec{k}^{\prime}\right)$ implies $\delta\left(\vec{k}_{1}+\vec{k}_{2}+\vec{k}_{3}+\vec{k}_{4}\right)$, the Dirac delta function associated with $q_{0}^{(4) s_{1} s_{2} s_{3} s_{4}}\left(\vec{k}_{1}, \vec{k}_{2}, \vec{k}_{3}, \vec{k}_{4}\right)$ in the first term of the above equation. For the second term, strong response occurs only when $s_{3}=-s_{1}, s_{4}=-s_{2}$ and $s^{\prime}=-s$. The dominant term is

(*) $\quad 2 \delta_{s^{\prime}-s} L_{k^{\prime}-k_{1}-k_{2}}^{s^{\prime}-s_{1}-s_{2}} q_{0}^{(2)-s_{1} s_{1}}\left(\vec{k}_{1}\right) q_{0}^{(2)-s_{2} s_{2}}\left(\vec{k}_{2}\right)\left(\pi \operatorname{sgnt} \delta\left(s_{1} \omega_{1}+s_{2} \omega_{2}-s \omega\right)+\mathrm{i} P \frac{1}{s_{1} \omega_{1}+s_{2} \omega_{2}-s \omega}\right)$.

Recall also $k^{\prime}+k=0$. For the third term, strong response occurs when $s_{3}=-s_{2}, s_{4}=s$ (any $s, s^{\prime}$ ); the dominant term is

(*) $\quad 2 L_{k_{1}-k_{2} k}^{s_{1}-s_{2} s} q_{0}^{(2)-s_{2} s_{2}}\left(\vec{k}_{2}\right) q_{0}^{(2) s s^{\prime}}\left(\vec{k}^{\prime}\right)\left(\pi \operatorname{sgnt} \delta\left(s_{1} \omega_{1}+s_{2} \omega_{2}-s \omega\right)+\mathrm{i} P \frac{1}{s_{1} \omega_{1}+s_{2} \omega_{3}-s \omega}\right)$.

For the fourth term, strong response occurs when $s_{3}=s, s_{4}=-s_{1}$ (any $\left.s, s^{\prime}\right)$; the dominant term is

(*) $\quad 2 L_{k_{2} k-k_{1}}^{s_{2}-s-s_{1}} q_{0}^{(2) s s^{\prime}}\left(k^{\prime}\right) q_{0}^{(2)-s s_{1}}\left(k_{1}\right)\left(\pi \operatorname{sgnt} \delta\left(s_{1} \omega_{1}+s_{2} \omega_{2}-s \omega\right)+\mathrm{i} P \frac{1}{s_{1} \omega_{1}+s_{2} \omega_{2}-s \omega}\right)$.

The only terms in $Q_{2}^{(2)} \mathrm{e}^{-\mathrm{i}\left(s \omega+s^{\prime} \omega^{\prime}\right) t}$ which contribute to $t$ growth arise from $F_{2}^{(2) s s^{\prime}}\left(k^{\prime}\right)$ and the three $(*)$ terms above. The terms containing $q_{0}^{(4) s_{1} s_{2} s_{3} s_{4}}$ do not contribute to secular growth. Thus, we do not require any assumption on the initial statistics other than the smoothness of the initial Fourier space cumulants or, equivalently, the decay of the physical space cumulants at large separations. We choose $F_{2}^{(2)}$ to kill the secular growth. 
For $s^{\prime}=-s$, we find

$$
\begin{aligned}
\frac{\mathrm{d} q_{0}^{s-s}(-\vec{k})}{\mathrm{d} t}= & \frac{\mathrm{d} q_{0}^{-s s}(\vec{k})}{\mathrm{d} t}=4 \pi \varepsilon^{2} \operatorname{sgnt} \sum_{s_{1} s_{2}} \int L_{k k_{1} k_{2}}^{s s_{1} s_{2}} q_{0}^{(2)-s s}(\vec{k}) q_{0}^{(2)-s_{1} s_{1}}\left(\vec{k}_{1}\right) q_{0}^{(2)-s_{2} s_{2}}\left(k_{2}\right) \\
& \times\left\{\frac{L_{-k-k_{1}-k_{2}}^{-s-s_{1}}}{q_{0}^{(2)-s s}(\vec{k})}+\frac{L_{k_{1} k-k_{2}}^{s_{1} s-s_{2}}}{q_{0}^{(2)-s_{1} s_{1}}\left(\vec{k}_{1}\right)}+\frac{L_{k_{2} k-k_{1}}^{s_{2} s-s_{1}}}{q_{0}^{(2)-s_{2} s_{2}}\left(\vec{k}_{2}\right)}\right\} \delta\left(s_{1} \omega_{1}+s_{2} \omega_{2}-s \omega\right) \delta\left(\vec{k}_{1}+\vec{k}_{2}-\vec{k}\right) \mathrm{d} \vec{k}_{1} \mathrm{~d} \vec{k}_{2} .
\end{aligned}
$$

Remark. The principal value terms cancel on the application of $\mathcal{P}^{00^{\prime}}=\mathcal{P}^{0-0}$. The Dirac delta terms add.

Eq. (2.19) is the kinetic equation for the redistribution of the spectral density $q_{0}^{(2)-s s}(\vec{k})$ via resonant exchange between the three waves $\vec{k}_{1}, \vec{k}_{2}, \vec{k}$ lying on the manifold $M$,

$$
s_{1} \omega\left(\vec{k}_{1}\right)+s_{2} \omega\left(\vec{k}_{2}\right)=s \omega(\vec{k}), \quad \vec{k}_{1}+\vec{k}_{2}=\vec{k}: M
$$

for some choices of $s_{1}, s_{2}, s$.

For $s^{\prime} \neq-s$ (thus, $s^{\prime}=s$ since $s^{2}=s^{\prime 2}=1$ ), we obtain

$$
\frac{\mathrm{d} q_{0}^{(2) s s^{\prime}}\left(\vec{k}^{\prime}\right)}{\mathrm{d} t}=\mathrm{i} \varepsilon^{2} q_{0}^{(2) s s^{\prime}}\left(\vec{k}^{\prime}\right)\left(\Omega_{k}^{s}+\Omega_{k^{\prime}}^{s^{\prime}}\right)
$$

where

$$
\begin{aligned}
\Omega_{k}^{s}= & 4 \sum_{s_{1} s_{2}} \int L_{k k_{1} k_{2}}^{s s_{1} s_{2}} L_{k_{1} k-k_{2}}^{s_{1} s-s_{2}} q_{0}^{(2)-s_{2} s_{2}}\left(\vec{k}_{2}\right) \delta_{0,12} \mathrm{~d} \vec{k}_{12} \\
& \times\left(P \frac{1}{s_{1} \omega_{1}+s_{2} \omega_{2}-s \omega}-\mathrm{i} \pi \operatorname{sgnt} \delta\left(s_{1} \omega_{1}+s_{2} \omega_{2}-s \omega\right)\right) .
\end{aligned}
$$

If we had included $L_{k k_{1} k_{2} k_{3}}^{s s_{1} s_{2}}$, then would be no change in (2.19) but

$$
\begin{aligned}
\Omega_{k}^{s} & =\sum_{s_{2}} \int \mathrm{d} \vec{k}_{2} q_{0}^{(2)-s_{2} s_{2}}\left(\vec{k}_{2}\right) G_{k k_{2}-k_{2} k}^{s s_{2}-s_{2} s} G_{k k_{2}-k_{2} k}^{s s_{2}-s_{2} s} \\
& =-3 \mathrm{i} L_{k k_{2}-k_{2} k}^{S s_{2}-s_{2} s}+4 \sum_{s_{1}} \int L_{k k_{2} k_{1}}^{s s_{2} s_{1}} L_{k_{1}-k_{2} k}^{s_{1}-s_{2} s}\left(P \frac{1}{W_{12,0}}-\mathrm{i} \pi \operatorname{sgnt} \delta\left(W_{12,0}\right)\right) \delta_{0,12} \mathrm{~d} \vec{k}_{1} .
\end{aligned}
$$

One can also show

$$
\frac{\mathrm{d} q_{0}^{(N) s s^{\prime} \cdots s^{(N-1)}}\left(\vec{k}^{\prime} \cdots \vec{k}^{(N-1)}\right)}{\mathrm{d} t}=\mathrm{i} \varepsilon^{2} q_{0}^{(N) s s^{\prime} \cdots s^{(N-1)}}\left(\vec{k}^{\prime} \cdots \vec{k}^{(N-1)}\right)\left(\Omega_{k}^{s}+\Omega_{k^{\prime}}^{s^{\prime}}+\cdots+\Omega_{k^{(N-1)}}^{s^{(N-1)}}\right) .
$$

The set of equations (2.21) and (2.24) can be jointly solved for all $N$ by the frequency renormalization

$$
s \omega_{k} \rightarrow s \omega_{k}+\varepsilon^{2} \Omega_{2 k}^{s}+\varepsilon^{4} \Omega_{4 k}^{s}+\cdots .
$$

It turns out that $\operatorname{Im} \Omega_{2 k}^{s}>0$ which means that the zeroth-order Fourier cumulants slowly decay due to resonances.

In summary, the asymptotic closure of the equations for wave (weak) turbulence occurs because (a) the linear dynamics causes phase mixing and a relaxation towards the state of joint Gaussianity on the time scale $1 / \varepsilon^{2} \omega_{0} \gg$ $t \gg 1 / \omega_{0}$, and (b) the nonlinear regeneration of cumulants of order $N$, on the time scale $1 / \varepsilon^{2} \omega_{0}$, which involves cumulants of order higher than $N$ and products of cumulants of order less than or equal to $N$, is dominated by the latter. 


\subsection{Properties of the kinetic equation}

We rewrite the kinetic equation (2.19) with $q_{0}^{-s s}(\vec{k})$ replaced by $n_{k}^{s}$ :

$$
\begin{aligned}
\frac{\mathrm{d} n_{k}^{s}}{\mathrm{~d} t}= & \varepsilon^{2} \text { sgnt } T[n]=4 \pi \varepsilon^{2} \operatorname{sgnt} \sum_{s_{1} s_{2}} \int L_{k k_{1} k_{2}}^{s s_{1} s_{2}} n_{k}^{s} n_{k_{1}}^{s_{1}} n_{k_{2}}^{s_{2}} \delta\left(\vec{k}_{1}+\vec{k}_{2}-\vec{k}\right) \delta\left(s_{1} \omega_{1}+s_{2} \omega_{2}-s \omega\right) \mathrm{d} \vec{k}_{1} \mathrm{~d} \vec{k}_{2} \\
& \times\left\{\frac{L_{-k-k_{1}-k_{2}}^{-s-s_{1}-s_{2}}}{n_{k}^{s}}+\frac{L_{k_{1} k-k_{2}}^{s_{1}-s_{2}}}{n_{k_{1}}^{s_{1}}}+\frac{L_{k_{2} k-k_{1}}^{s_{2}-s_{1}}}{n_{k_{2}}^{s_{2}}}\right\} .
\end{aligned}
$$

We list its properties:

1. The mechanism for energy transfer is one of resonance.

2. The property $L_{k_{1} k-k_{2}}^{s_{1} s-s_{2}}=\left(s_{1} / s\right) L_{k k_{1} k_{2}}^{s s_{2}}=-\left(s_{1} / s\right) L_{-k-k_{1}-k_{2}}^{-s-s_{1}-s_{2}}$ shows that $n_{k}^{s}=T / \omega_{k}$, the thermodynamic equilibrium or Rayleigh-Jeans spectrum of energy equipartition (recall that $\sum_{s} \omega_{k} n_{k}^{s}$ is the spectral energy density), is an exact solution of (2.26). This stationary solution has a zero flux of energy. As a result, it is not particularly relevant for non-equilibrium situations. Indeed, since in most applications there is a dissipative sink at $\omega_{k}=\infty$, the effective temperature $T$ of the random wavefield is zero.

3. Conservation of energy $E=(1 / 2) \sum_{s} \int \omega_{k} n_{k}^{s} \mathrm{~d} \vec{k}$ follows from multiplying (2.26) by $\frac{1}{2} \omega_{k}$, summing over $s$ and integrating over $\vec{k}$ and then interchanging the order of integration. Formally, in the second (third) term in (2.26), interchange $\vec{k}_{1}\left(\vec{k}_{2}\right)$ and $\vec{k}, s_{1}\left(s_{2}\right)$ and $s$, and change the sign of both $s_{2}$ and $k_{2}\left(s_{1}\right.$ and $\left.\vec{k}_{1}\right)$. Using the properties (1)-(4) of Section 2.1, we find the integrand of (2.26) becomes $L_{k k_{1} k_{2}}^{s s_{1} s_{2}} L_{-k-k_{1}-k_{2}}^{-s-s_{2}-s_{2}} n_{k}^{s} n_{k_{1}}^{s_{1}} n_{k_{2}}^{s_{2}} \delta_{12,0} \delta\left(W_{12,0}\right)\left\{\omega_{k}-\right.$ $\left.\left(s_{1} / s\right) \omega_{1}-\left(s_{2} / s\right) \omega_{2}\right\}$ which vanishes on the resonant manifold.

But this result is only formal and the exchange of integration order relies on Fubini's theorem which demands that the double integral (one can integrate out $\vec{k}_{2}$ ) converges before any exchange of order is done. We must check that $n_{k}^{s}$ has the right behavior in $\vec{k}$ to allow this. It may turn out that convergence fails after a certain time $t^{*}<\infty$ because the solution $n_{k}^{s}(t)$ reaches a stationary state in finite time which renders the $\vec{k}$ integration of the collision integral (the right-hand side of (2.26)) divergent. It also turns out that the stationary state is no longer energy conserving. What happens is that energy can be lost to $k=\infty$.

4. Reversibility $[11,12]$. We have shown that if the Fourier space cumulants are initially smooth, say at some time $t=t_{0}$, then $n_{k}^{s}$ evolves according to (2.26) with sgnt replaced by $\operatorname{sgn}\left(t-t_{0}\right)$. The graph versus time of $n_{k}^{s}$ is shown in Fig. 3. We note that while the detailed slope of $q^{-s s}(k, t)$ will be continuous, the slope of $n_{k}^{s}(t)$ at $t=t_{0}$ (found after averaging $q^{-s s}(k, t)$ over all oscillations) is discontinuous. There is nothing unusual or surprising in this; the system will flow towards some attracting equilibrium state no matter whether it goes forward or backwards in time. The loss of exact reversibility is the result of a loss of phase information introduced in the mathematical formulation through the limit $\omega_{0}\left(t-t_{0}\right) \rightarrow \infty, \varepsilon^{2} \omega_{0}\left(t-t_{0}\right)$ finite. However, this result opens the door to an apparent contradiction. Suppose one were to redo the calculation beginning at a later time

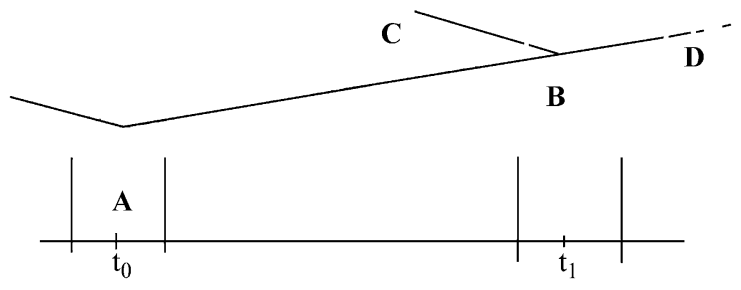

Fig. 3. The solution $\eta(\vec{k}, t)$ is retraceable. 
$t_{1}, \omega_{0}\left(t_{1}-t_{0}\right)=\mathrm{O}\left(\varepsilon^{-2}\right)$. Then (2.26) would suggest that the slope of $n_{k}^{s}$ at $t_{1}$ would mirror that at $t_{0}$ where there is a discontinuity in slope. Namely, in (2.26), $\operatorname{sgn}\left(t-t_{0}\right)$ (which is +1 for $t>t_{0}$ ) would be replaced by $\operatorname{sgn}\left(t-t_{1}\right)$. It would be impossible to retrace the solution $n_{k}^{s}$ from $t_{1}$ towards $t_{0}$. But this conclusion is false. The reason is that, over long times, long-distance correlations are built up and the Fourier transform of $R^{(N)}$ picks up a non-smooth component at order $\varepsilon^{(N-2)}$. In particular, $Q^{(3) s s^{\prime} s^{\prime \prime}}\left(\vec{k}^{\prime}, \vec{k}^{\prime \prime}\right)$ picks up an order $\varepsilon$ non-smooth behavior given by (2.17). Taking account of the fact that at $t_{1}, Q^{(3)}$ has this order $\varepsilon$ behavior leads to additional terms in the kinetic equation when one calculates beginning at $t_{1}$. They are exactly equal to (2.26) with the $\operatorname{sgn}\left(t-t_{0}\right)$ (which because $t_{1}>t_{0}$ is +1$)$ replaced by $\left(1-\operatorname{sgn}\left(t-t_{1}\right)\right.$ ). The effect of the non-smoothness in $Q^{(3)}$ does not change (2.26) at all for $t>t_{1}$. For $t<t_{1}$, on the other hand, it changes the evolution along BC (see Fig. 3) (because of $\left.\operatorname{sgn}\left(t-t_{1}\right)\right)$ to one along BA and BD (because $\operatorname{sgn}\left(t-t_{1}\right)+1-\operatorname{sgn}\left(t-t_{1}\right)=1=\operatorname{sgn}\left(t-t_{0}\right)$ ). The solution $n_{k}^{s}\left(T=\varepsilon^{2}\left(t-t_{0}\right)\right)$ can be retraced on the $\varepsilon^{-2} \omega_{0}^{-1}$ time scale. There is no inconsistency or paradox.

5. Finite flux spectrum. The realization that the kinetic equation (2.26) has attracting, stationary solutions which describe a constant flux of conserved densities is one of the many important contributions of Zakharov. Before we give his derivation, we shall first deduce the main result from dimensional considerations. We have seen that there is, at least locally in time, energy conservation. We can then write $E_{k}=(1 / 2) \sum_{s} \omega_{k} n_{k}^{s}$ as the $\vec{k}$ divergence of an energy flux $\vec{P}_{k}$. Assuming isotropy and angle averaging, we find (take $t>0$ )

$$
\frac{\partial}{\partial t} E_{k} k^{(d-1)}=\varepsilon^{2} \sum_{s} \omega_{k} k^{(d-1)} T[n]=-\varepsilon^{2} \frac{\partial P}{\partial k} .
$$

Assuming $n_{k}=c P^{1 / 2} k^{-x}$, we have that the $k$ dependence of $\omega_{k} k^{(d-1)} T[n]$ is $k^{\alpha} \cdot k^{(d-1)} \cdot k^{2 \beta} \cdot c^{2} P k^{-2 x} k^{-\alpha} k^{d}\left(\omega_{k} \sim\right.$ $k^{\alpha}, L^{2} \sim k^{2 \beta}, n^{2} \sim c^{2} P k^{-2 x}, \delta(\omega) \sim k^{-\alpha}, \mathrm{d} \vec{k} \sim k^{d}$ and this should equal $P k^{-1}$. Solving, we find $x=\beta+d$. The spectrum

$$
n_{k}^{s}=c P^{1 / 2} k^{-(\beta+d)}
$$

has two very interesting properties.

First, if we calculate the total energy $E=(1 / 2) \sum_{s} \int \omega_{k} n_{k} \mathrm{~d} \vec{k}$, we find this behaves as $c P^{1 / 2} \int^{\infty} k^{(\alpha-\beta)}(\mathrm{d} k / k)$. Now imagine that this spectrum is responsible for carrying a finite flux of energy, inserted at a steady rate at some low value of $k$, say $k_{\mathrm{L}}$. Clearly, the spectrum around $k_{\mathrm{L}}$ will not be universal but for $k \gg k_{\mathrm{L}}$, let us imagine it is and approaches (2.28). For $\beta>\alpha$, the energy integral converges. In this case, the spectrum (2.28), if it is indeed the attractor, can only absorb a finite amount of energy from the source. We say it has finite capacity. This has several consequences. If energy is delivered to the system at a finite rate and the universal finite flux (Kolmogorov) spectrum can only absorb a finite amount, then there must be a sink at $k=\infty$ to absorb that energy. Moreover, the front (imagine the initial spectrum of $n_{k}^{s}$ is on finite support, e.g. $\left.n_{k}^{s}(t=0)=0, k>k_{0}\right)$ that sets up the spectrum (2.28) must reach $k=\infty$ in a finite time $t^{*}$ equal to the time it takes the source energy to fill the finite capacity spectrum (2.28). This means that in the absence of a sink at $k=\infty$, the "inviscid" system must develop highly irregular behavior at large $k$. This observation has not yet been exploited in the investigation of possible singularity development in the long-time behavior of nonlinear PDEs of conservative type. For $\beta \leq \alpha$, the spectrum (2.28) has infinite capacity and can absorb whatever energy is fed into it. In this case, the front that sets up (2.28) from spectrum initially on finite support, reaches $k=\infty$ in infinite time. We will examine these fronts in Section 5. There are anomalies and surprises!

Second, let us examine the ratio of the linear $\left(t_{\mathrm{L}}\right)$ and nonlinear $\left(t_{\mathrm{NL}}\right)$ time scales on the spectrum (2.28) as a function of $k$. 
Estimating $t_{\mathrm{L}}^{-1}$ by $\omega_{k}$ and $t_{\mathrm{NL}}^{-1}$ as $\left(1 / n_{k}\right) \partial n_{k} / \partial t$, we find (we take $n_{k}^{+}=n_{k}^{-}$),

$$
\frac{t_{\mathrm{L}}}{t_{\mathrm{NL}}}=\frac{1}{\omega_{k} n_{k}} \frac{\partial n_{k}}{\partial t}=\frac{\varepsilon^{2}}{\omega_{k} n_{k} k^{(d-1)}} \frac{\partial P}{\partial k} .
$$

The last term behaves as $k^{-\alpha} \cdot P^{-1 / 2} k^{(\beta+d)} \cdot k^{(1-d)} \cdot \varepsilon^{2} P k^{-1}=\varepsilon^{2} P^{1 / 2} k^{(\beta-2 \alpha)}$. For $\beta>2 \alpha(\beta<2 \alpha)$, the ratio of linear to nonlinear time scales, which for the applicability of the weak turbulence approximation, must be small (because of the need to separate scales), is no longer uniformly bounded by $\varepsilon^{2}$. It diverges for large (small) $k$ suggesting that when either $\beta \gtrless 2 \alpha$, the small (large) scale behavior of the system becomes more and more fully nonlinear. This finding will be corroborated by a separate calculation on the validity of the weak turbulence approximation carried out in Section 4. There we will find that the ratio of the structure function $S_{N}(r)=$ $\left\langle\left(v^{s}(\vec{x}+\vec{r})-v(\vec{x})\right)^{N}\right\rangle$ to $\left(S_{2}\right)^{(N / 2)}$ is a finite series in powers of $\varepsilon^{2} P^{1 / 2} r^{(2 \alpha-\beta)}$ which is not uniformly bounded at small scales when $\beta>2 \alpha$. Moreover, the coefficients grow with $N$. Similarly, for large $r$, the ratios of cumulants $R^{(N)}$ to $\left(R^{(2)}\right)^{N / 2}$ diverge when $\beta<2 \alpha$.

Note: When we evaluate the structure functions on the finite energy flux spectrum, we choose $v^{s}(x)$ to be the physical field whose pair correlation has the spectral energy $\omega_{k} n_{k}$ as its Fourier transform. The formal Fourier transform of $v^{s}(\vec{x})$ is therefore $\sqrt{\omega_{k}} A_{\vec{k}}^{s}$.

For surface tension waves, $\beta=\frac{9}{4}, \alpha=\frac{3}{2}, d=2$, so that $n_{k}=c P^{1 / 2} k^{-17 / 4}$. Since $2 \alpha>\beta>\alpha$, we find that the spectrum has finite capacity and also preserves the uniformity of the wave turbulence approximation at high $k$.

We now turn to the Zakharov derivation of (2.28). Using property 3 of Section 2.1 repeatedly and assuming $n_{k}^{s}=n_{k}, s=+,-$, we rewrite (2.26) as

$$
\frac{\mathrm{d} n_{k}}{\mathrm{~d} t}=4 \pi \varepsilon^{2} \int\left|L_{k k_{1} k_{2}}^{+++}\right|^{2} \delta\left(k_{1}+k_{2}-k\right) \mathrm{d} \vec{k}_{1} \mathrm{~d} \vec{k}_{2} n_{k} n_{k_{1}} n_{k_{2}} F\left(\omega, \omega_{1}, \omega_{2}\right),
$$

where

$$
\begin{aligned}
F\left(\omega, \omega_{1}, \omega_{2}\right)= & \left(\frac{1}{n}-\frac{1}{n_{1}}-\frac{1}{n_{2}}\right) \delta\left(\omega-\omega_{1}-\omega_{2}\right)+\left(\frac{1}{n}-\frac{1}{n_{1}}+\frac{1}{n_{2}}\right) \delta\left(\omega_{1}-\omega-\omega_{2}\right) \\
& +\left(\frac{1}{n}+\frac{1}{n_{1}}-\frac{1}{n_{2}}\right) \delta\left(\omega_{2}-\omega-\omega_{1}\right),
\end{aligned}
$$

where $n_{k}=n, n_{k_{1}}=n_{1}, n_{k_{2}}=n_{2}$. Assuming isotropy, writing $n, n_{1}, n_{2}$ as functions of $\omega, \omega_{1}$ and $\omega_{2}$, respectively, and introducing $N_{\omega}$ by $\int_{0}^{\infty} N_{\omega} \mathrm{d} \omega=\int n_{k} \mathrm{~d} \vec{k}$, we find upon averaging over angles in $\vec{k}_{1}, \vec{k}_{2}$ space (denoted by \langle\rangle ),

$$
\frac{\mathrm{d} N_{\omega}}{\mathrm{d} t}=S[n]=\int_{\Delta} S_{\omega \omega_{1} \omega_{2}} n n_{1} n_{2} F\left(\omega, \omega_{1}, \omega_{2}\right) \mathrm{d} \omega_{1} \mathrm{~d} \omega_{2},
$$

where $\Delta$ is the quarter plane $\omega_{1}, \omega_{2}>0$ and

$$
S_{\omega \omega_{1} \omega_{2}}=4 \pi \varepsilon^{2}\left\langle\left|L_{k k_{1} k_{2}}^{+++}\right|^{2} \delta\left(\vec{k}_{1}+\vec{k}_{2}-\vec{k}\right)\right\rangle\left(k k_{1} k_{2}\right)^{(d-1)} \frac{\mathrm{d} k}{\mathrm{~d} \omega} \frac{\mathrm{d} k_{1}}{\mathrm{~d} \omega_{1}} \frac{\mathrm{d} k_{2}}{\mathrm{~d} \omega_{2}} .
$$

If $k=\omega^{1 / \alpha}$ and $L$ is homogeneous of degree $\beta$, then $S$ is homogeneous of degree $\tau=2((\beta+d) / \alpha)-3$. (Note: A correction $\delta$ must be added to $\tau$ when the waves are almost non-dispersive; see [32].)

We want to find stationary solutions to (2.30) other than the thermodynamic equilibrium $\omega_{k} n_{k}=T$ which makes $F$ identically zero. The class of solutions which Zakharov discovered in the late 1960s makes use of the scaling symmetries (homogeneity) in both the dispersion relation and the coefficients $L_{k k_{1} k_{2}}^{+++}$and $S_{\omega \omega_{1} \omega_{2}}$. The idea is to find 
a transformation (now called the Zakharov transformation) which maps the lines $\omega_{1}+\omega_{2}=\omega, \omega_{1}=\omega+\omega_{2}$ and $\omega_{2}=\omega+\omega_{1}$ on which $F$ is supported into each other. This is achieved by the maps

$$
\omega_{1} \rightarrow \frac{\omega^{2}}{\omega_{1}}, \quad \omega_{2} \rightarrow \frac{\omega \omega_{2}}{\omega_{1}},
$$

which sends $\omega_{1}=\omega+\omega_{2}$ into $\omega=\omega_{1}+\omega_{2}$ and

$$
\omega_{1} \rightarrow \frac{\omega \omega_{1}}{\omega_{2}}, \quad \omega_{2} \rightarrow \frac{\omega^{2}}{\omega_{2}},
$$

which sends $\omega_{2}=\omega+\omega_{1}$ into $\omega=\omega_{1}+\omega_{2}$. Next, let $n=c \omega^{-x}$ for values of $x$ for which the collision integral $S[n]$ exists. This should be checked a posteriori. There is a danger otherwise that divergences in $S[n]$ could be cancelled by application of the Zakharov transformation.

$$
S\left[n=c \omega^{-x}\right]=c^{2} \int_{\Delta} S_{\omega \omega_{1} \omega_{2}}\left(\omega \omega_{1} \omega_{2}\right)^{-x} \hat{F}\left(\omega, \omega_{1}, \omega_{2}\right) \mathrm{d} \omega_{1} \mathrm{~d} \omega_{2},
$$

where $\hat{F}=\left(\omega^{x}-\omega_{1}^{x}-\omega_{2}^{x}\right) \delta\left(\omega-\omega_{1}-\omega_{2}\right)+\left(\omega^{x}-\omega_{1}^{x}+\omega_{2}^{x}\right) \delta\left(\omega_{1}-\omega-\omega_{2}\right)+\left(\omega^{x}+\omega_{1}^{x}-\omega_{2}^{x}\right) \delta\left(\omega_{2}-\omega-\omega_{1}\right)$. Apply (2.31) to the second term and (2.32) to the third and use the facts that $S_{\omega\left(\omega^{2} / \omega_{1}\right)\left(\omega \omega_{2} / \omega_{1}\right)}=\left(\omega / \omega_{1}\right)^{\tau} S_{\omega_{1} \omega \omega_{2}}=$ $\left(\omega / \omega_{1}\right)^{\tau} S_{\omega \omega_{1} \omega_{2}}$ and the Jacobian of $(2.31)$ is $\left(\omega / \omega_{1}\right)^{3}$ to rewrite $S[n]$ as

$$
S[n]=c^{2} \int S_{\omega \omega_{1} \omega_{2}}\left(\omega \omega_{1} \omega_{2}\right)^{-x}\left(\omega^{x}-\omega_{1}^{x}-\omega_{2}^{x}\right)\left(1-\left(\frac{\omega_{1}}{\omega}\right)^{y}-\left(\frac{\omega_{2}}{\omega}\right)^{y}\right) \delta\left(\omega-\omega_{1}-\omega_{2}\right) \mathrm{d} \omega_{1} \mathrm{~d} \omega_{2},
$$

where $y=2 x-\tau-2=2 x-2((\beta+d) / \alpha)+1$. Using homogeneity we may also write $S[n]$ as

$$
S[n]=c^{2} \omega^{(\tau-2 x+1)} I(x, y)=c^{2} \omega^{(-y-1)} I(x, y),
$$

where $\omega_{1}=\xi \omega, \omega_{2}=\eta \omega$ and

$$
I(x, y)=c^{2} \int S_{1 \xi \eta}(\xi \eta)^{-x}\left(1-\xi^{x}-\eta^{x}\right)\left(1-\xi^{y}-\eta^{y}\right) \delta(1-\xi-\eta) \mathrm{d} \xi \mathrm{d} \eta
$$

is the line integral along $\xi+\eta=1$ between $(\xi=0, \eta=1)$ and $(\xi=1, \eta=0)$. Observe that $S[n]=0$ both for

$$
\begin{aligned}
& x=1 \quad \text { Rayleigh-Jeans, } \\
& y=1 \quad \text { or } \quad x=\frac{\beta+d}{\alpha} \quad \text { pure Kolmogorov. }
\end{aligned}
$$

The constant $c$ may be determined using first part of (2.31) and defining $P$ such that

$$
\frac{\partial \omega N_{\omega}}{\partial t}=\omega S[n]=-\frac{\partial P}{\partial \omega},
$$

and integrating to find

$$
-P=c^{2} \frac{\omega^{(-y+1)}}{-y+1} I(x, y)
$$

Taking the limit of $y \rightarrow 1$, we find

$$
P=\left.c^{2} \frac{\partial I}{\partial y}\right|_{y=1}
$$


or

$$
c=P^{1 / 2}\left(\frac{\partial I}{\partial y}\right)^{-1 / 2} .
$$

The ranges of convergence of $S[n]$ (before making the Zakharov transformation) and $I(x, y)$, and $\partial I / \partial y$ must be checked to establish consistency. The stability of these solutions to both isotropic and non-isotropic perturbations should also be examined. The reader should refer to Refs. [25-27,29,41,43].

\section{Four-wave resonances}

Suppose the manifold $M_{3}$ for three-wave resonances is null, either because the dispersion relation is such that one cannot find triads $\vec{k}, \vec{k}_{1}, \vec{k}_{2}$ satisfying (2.12) (e.g. gravity water waves $\omega=\sqrt{g k}$ ) or because the coefficients $L_{k k_{1} k_{2}}^{s s_{1} s_{2}}$ are identically zero on $M_{3}$. In either case, there is no redistribution of energy on the $\varepsilon^{-2} \omega_{0}^{-1}$ time scale. There is, however, still the frequency renormalization (at order $\varepsilon^{2}$ ) with the delta function missing. Continuing the expansions to higher order in $\varepsilon$ (it is convenient to transform $A_{k}^{s}$ to $B_{k}^{s}+\varepsilon F_{1}\left[B_{k}^{s}\right]+\cdots$ so as to eliminate the quadratic terms in (2.2); in Hamiltonian systems, this choice can be made canonical), we find that a non-trivial closure for spectral particle density $n_{k}^{s}$ occurs at $\mathrm{O}\left(\varepsilon^{4}\right)$ and is given by

$$
\begin{aligned}
\frac{\mathrm{d} n_{k}^{s}}{\mathrm{~d} t}= & 12 \pi \varepsilon^{4} \sum_{s_{1} s_{2} s_{3}} \int G_{k k_{1} k_{2} k_{3}}^{s s_{1} s_{2} s_{3}} n_{k}^{s} n_{k_{1}}^{s_{1}} n_{k_{2}}^{s_{2}} n_{k_{3}}^{s_{3}} \delta\left(\vec{k}_{1}+\vec{k}_{2}+\vec{k}_{3}-\vec{k}\right) \\
& \times\left\{\frac{G_{-k-k_{1}-k_{2}-k_{3}}^{-s-s_{1}-s_{2}-s_{3}}}{n_{k}^{s}}+\stackrel{123}{\mathcal{P}} \frac{G_{k_{1} k-k_{2}-k_{3}}^{s_{1}-s_{2}-s_{3}}}{n_{k_{1}}^{s_{1}}}\right\} \delta\left(s_{1} \omega_{1}+s_{2} \omega_{2}+s_{3} \omega_{3}-s \omega\right) \mathrm{d} \vec{k}_{1} \mathrm{~d} \vec{k}_{2} \mathrm{~d} \vec{k}_{3} .
\end{aligned}
$$

The manifold $M_{4}$ is

$$
\vec{k}_{1}+\vec{k}_{2}+\vec{k}_{3}=\vec{k}, \quad s_{1} \omega_{1}+s_{2} \omega_{2}+s_{3} \omega_{3}=s \omega .
$$

The coupling coefficient $G_{k k_{1} k_{2} k_{3}}^{s s_{1} s_{2} s_{3}}$ is given by

$$
G_{k k_{1} k_{2} k_{3}}^{s s_{1} s_{2} s_{3}}=L_{k k_{1} k_{2} k_{3}}^{s s_{1} s_{2} s_{3}}-\frac{2 \mathrm{i}}{3} \stackrel{123}{\mathcal{P}} \sum_{s_{4}} \frac{L_{k k_{2}+k_{3}+k_{1}}^{s-s_{4} s_{1}} L_{k_{2}+k_{3} k_{2} k_{3}}^{-s_{4} s_{2} s_{3}}}{s_{2} \omega_{2}+s_{3} \omega_{3}+s_{4} \omega\left(\vec{k}_{2}+\vec{k}_{3}\right)} .
$$

The frequency renormalization is

$$
s \omega_{k} \rightarrow s \omega_{k}+\varepsilon^{2} \Omega_{k}^{s}+\varepsilon^{4} \Gamma_{k}^{s}+\cdots,
$$

where $\Omega_{k}^{s}$ is as given in (2.23) without the $\delta\left(s_{1} \omega_{1}+s_{2} \omega_{2}-s \omega\right)$ terms. $\Gamma_{k}^{s}$ contains a positive imaginary component proportional to $\delta\left(s_{1} \omega_{1}+s_{2} \omega_{2}+s_{3} \omega_{3}-s \omega\right)$ indicating that even the leading-order contributions to the Fourier space cumulants of order $N>2$ decay over long times. Remember, however, that their physical space counterparts decay much more quickly due to the time oscillating factors in the integrand of the Fourier transform.

In this lecture, we will concentrate on systems given by the Hamiltonian

$$
\mathcal{H}=\int \omega_{k} A_{k}^{*} A_{k} \mathrm{~d} \vec{k}+\frac{1}{2} \int T_{k k_{1}, k_{2} k_{3}} A_{k}^{*} A_{k_{1}}^{*} A_{k_{2}} A_{k_{3}} \delta_{01,23} \mathrm{~d} \vec{k}_{0123}
$$

for which we identify $A_{k}$ with $A_{k}^{+}, A_{k}^{*}$ with $A_{k}^{-}$and set $n_{k}^{+}=n_{k}^{-}$and take

$$
L_{k k_{1} k_{2} k_{3}}^{s s_{1} s_{2} s_{3}}=\frac{1}{3} \mathrm{i} s \stackrel{123}{\mathcal{P}} \delta_{s_{1}-s} \delta_{s_{2} s} \delta_{s_{3} s} T_{k-k_{1}, k_{2} k_{3}}
$$


where $\delta_{s_{1} s_{2}}$ is the Kronecker delta. In the product $L_{k k_{1} k_{2} k_{3}}^{s s_{1} s_{2} s_{3}} L_{-k-k_{1}-k_{2}-k_{3}}^{-s-s_{1}-s_{2}-s_{3}}$ only three out of the possible nine terms survive so that the factor 12 in (3.1) becomes 4 .

$$
\begin{aligned}
\frac{\mathrm{d} n_{k}}{\mathrm{~d} t}= & T[n]=4 \pi \varepsilon^{4} \int\left|T_{k k_{1}, k_{2} k_{3}}\right|^{2} n n_{1} n_{2} n_{3} \\
& \times\left(\frac{1}{n}+\frac{1}{n_{1}}-\frac{1}{n_{2}}-\frac{1}{n_{3}}\right) \delta\left(\omega+\omega_{1}-\omega_{2}-\omega_{3}\right) \delta\left(\vec{k}+\vec{k}_{1}-\vec{k}_{2}-\vec{k}_{3}\right) \mathrm{d} \vec{k}_{123} .
\end{aligned}
$$

We can also rewrite (3.6) as

$$
\frac{\mathrm{d} n_{k}}{\mathrm{~d} t}=4 \pi \varepsilon^{4} \int\left|T_{k k_{1}, k_{2} k_{3}}\right|^{2} H\left(n, n_{1}, n_{2}, n_{3}\right) \delta\left(\omega+\omega_{1}-\omega_{2}-\omega_{3}\right) \delta\left(\vec{k}+\vec{k}_{1}-\vec{k}_{2}-\vec{k}_{3}\right) \mathrm{d} \vec{k}_{123},
$$

where

$$
H\left(n, n_{1}, n_{2}, n_{3}\right)=n_{2} n_{3}\left(n+n_{1}\right)-n n_{1}\left(n_{2}+n_{3}\right) .
$$

If $A_{k}$ had been an operator obeying Bose statistics rather than a complex generalized function, corresponding to a classical wave field, then we would find the same equation for the particle density of bosons with

$$
H\left(n, n_{1}, n_{2}, n_{3}\right)=n_{2} n_{3}(1+n)\left(1+n_{1}\right)-n n_{1}\left(1+n_{2}\right)\left(1+n_{3}\right) .
$$

If $A_{k}$ had been an operator obeying Fermi statistics, then

$$
H\left(n, n_{1}, n_{2}, n_{3}\right)=n_{2} n_{3}(1-n)\left(1-n_{1}\right)-n n_{1}\left(1-n_{2}\right)\left(1-n_{3}\right) .
$$

The particle equation for fermions can be understood as a Boltzmann equation for two particle collisions with momenta and energies equal to $\vec{k}, \vec{k}_{1}, \omega, \omega_{1}$ before and $\vec{k}_{2}, \vec{k}_{3}, \omega_{2}, \omega_{3}$ after. The particle densities $n, n_{1}, n_{2}, n_{3}$ represent the probabilities of finding a particle in states $(\vec{k}, s \omega),\left(k_{j}, s_{j} \omega_{j}\right)_{j=1}^{3}$ and their complements $1-n, 1-$ $n_{1}, 1-n_{2}, 1-n_{3}$ represent the probabilities that the states are vacant. Recall that the Pauli exclusion principle only allows transfer to vacant states.

Eqs. (3.1) and (3.6) have two conserved quantities

$$
\begin{aligned}
& N=\int n_{k} \mathrm{~d} \vec{k}=\int_{0}^{\infty} N_{\omega} \mathrm{d} \omega, \\
& E=\int \omega_{k} n_{k} \mathrm{~d} \vec{k}=\int_{0}^{\infty} \omega N_{\omega} \mathrm{d} \omega
\end{aligned}
$$

for all three systems (3.8)-(3.10). In general, the original equation (2.1) may not formally conserve total particle number. However, when three-wave interactions are absent and (3.1) obtains, we find that particle number is conserved as long as the coupling coefficient $G$ vanishes whenever the set $\left(-s, s_{1}, s_{2}, s_{3}\right)$ does not consist of two pluses and two minuses. It may or may not be conserved at higher orders. The formal proof for conservation of $N$ and $E$ relies on the exchange of orders of integration, the validity of which has already been discussed in Section 2.7. Similar qualifications apply here. Each conservation law may only obtain for a finite time. Connected with these conserved quantities are the thermodynamic equilibria. For classical waves,

$$
\frac{1}{n_{k}}=\frac{1}{T}\left(\omega_{k}-\mu\right),
$$

represents a combination of equipartition of energy $(\mu=0)$ and particles $(T \rightarrow \infty, \mu / T$ finite), the Rayleigh-Jeans spectrum. For bosons, the corresponding spectrum is the Bose-Einstein distribution, 


$$
\frac{1}{n_{k}}=\exp \frac{1}{T}\left(\omega_{k}-\mu\right)-1
$$

which tends to (3.10) in the high temperature $(T)$ limit. For fermions, there is the Fermi-Dirac distribution

$$
\frac{1}{n_{k}}=\exp \frac{1}{T}\left(\omega_{k}-\mu\right)+1
$$

The parameter $T$ is usually called temperature and $\mu$ the chemical potential.

For all of these solutions, the fluxes of particles and energy across the spectrum are zero. Therefore, in order to find solutions of (3.6) for non-equilibrium situations which carry finite fluxes of particles and energy, we must seek a richer class of stationary solutions. Zakharov was the first to find these and to point out that they were far more relevant in applications than the thermodynamic equilibria. We will not give details of the derivation here. For these, we point the reader to Refs. [32] or [33]. We will, however, discuss the results in some detail because the presence of an extra conserved density (particles) has important consequences.

Assuming isotropy and defining $N_{\omega}=\Omega_{0} k^{(d-1)}(\mathrm{d} k / \mathrm{d} \omega) n_{k}(\omega), \Omega_{0}$ is the solid angle in $d$ dimensions, we find after angle averaging that

$$
\frac{\mathrm{d} N_{\omega}}{\mathrm{d} t}=\int_{\Delta} S_{\omega \omega_{1} \omega_{2} \omega_{3}} n n_{1} n_{2} n_{3}\left(\frac{1}{n}+\frac{1}{n_{1}}-\frac{1}{n_{2}}-\frac{1}{n_{3}}\right) \delta\left(\omega+\omega_{1}-\omega_{2}-\omega_{3}\right) \mathrm{d} \omega_{1} \mathrm{~d} \omega_{2} \mathrm{~d} \omega_{3},
$$

where $\Delta$ is the region $\omega_{2}>0, \omega_{2}+\omega_{3}>\omega, \omega_{3}>0$ in the $\omega_{2}, \omega_{3}$-plane. If $T_{k k_{1}, k_{2} k_{3}}$ is homogeneous of degree $\gamma, S_{\omega \omega_{1} \omega_{2} \omega_{3}}$ is homogeneous of degree $\sigma=((2 \gamma+3 d) / \alpha)-4$. Because $N_{\omega}$ and $E_{\omega}=\omega N_{\omega}$ are conserved densities, we may write

$$
\frac{\mathrm{d} N_{\omega}}{\mathrm{d} t}=\frac{\partial Q}{\partial \omega}, \quad \frac{\mathrm{d} \omega N_{\omega}}{\mathrm{d} t}=-\frac{\partial P}{\partial \omega}
$$

with $P(Q)$ positive when energy flows to higher (lower) frequencies $\omega$. As before in Section 2.7, we can find the pure Kolmogorov spectra,

$$
\begin{aligned}
& n_{k}=c_{1} Q^{1 / 3} k^{((-2 \gamma+3 d) / 3)+\alpha / 3}, \\
& n_{k}=c_{2} P^{1 / 3} k^{-(2 \gamma+3 d) / 3}
\end{aligned}
$$

for which $c_{1}$ and $c_{2}$ are calculable constants. Equilibrium (3.18) and (3.19) carries a finite particle (energy) flux and zero energy (particle) flux. For deep ocean water waves, $\gamma=3, d=2, \alpha=\frac{1}{2}$ so that for large $k, n_{k}=c_{2} P^{1 / 3} k^{-4}$ or $\omega_{k} n_{k}=c_{2} P^{1 / 3} k^{-7 / 2}$.

Only in certain cases, however, will the pure finite flux solutions be relevant. To see this, imagine a situation depicted in Fig. 4 in which particles and energy are added to the system at frequency $\omega_{0}$ at the rates $Q_{0}$ and $P_{0}=\omega_{0} Q_{0}$, respectively.

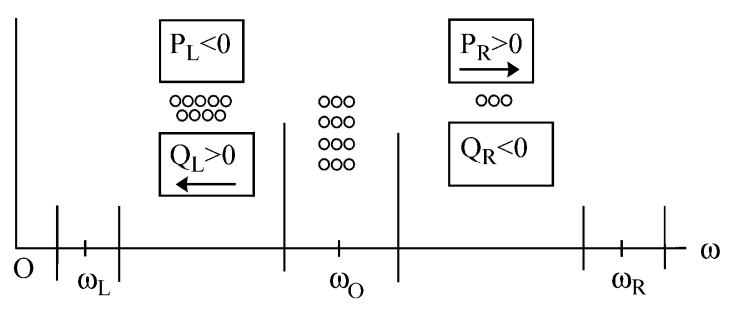

Fig. 4. Left and right fluxes of particles and energy from a source at $\omega_{0}$ to sinks at $\omega_{\mathrm{L}}$ and $\omega_{\mathrm{R}}$. 
Suppose there are no leakages of particles or energy at $\omega=0, \infty$. Then the rate of particle and energy input $Q_{0}$ and $P_{0}=\omega_{0} Q_{0}$ must be balanced by dissipation $Q_{\mathrm{d}}$ and $P_{\mathrm{d}}$ in order that a steady state is achieved. Conservation implies that the average frequency of pumping $\omega_{0}=P_{0} / Q_{0}$ and damping $\omega_{\mathrm{d}}=P_{\mathrm{d}} / Q_{\mathrm{d}}$ must be equal. Therefore, if $\omega_{\mathrm{d}} \neq \omega_{0}$, namely if one of the sinks is near $\omega=\infty$, there must be two sinks. We will put them at $\omega_{\mathrm{L}}$ and $\omega_{\mathrm{R}}$. Equating flux rates $P_{\mathrm{R}}-P_{\mathrm{L}}=P_{0}=\omega_{0} Q_{0}$ and $Q_{\mathrm{L}}-Q_{\mathrm{R}}=Q_{0}$, and assuming that all particles and energies traveling through the left and right windows of transparency are absorbed at $\omega_{\mathrm{L}}$ and $\omega_{\mathrm{R}}$, respectively, we have that $P_{\mathrm{L}}=-\omega_{\mathrm{L}} Q_{\mathrm{L}}$ and $P_{\mathrm{R}}=-\omega_{\mathrm{R}} Q_{\mathrm{R}}$. Therefore, we can calculate

$$
\begin{aligned}
& Q_{\mathrm{L}}=Q_{0} \frac{\omega_{\mathrm{R}}-\omega_{0}}{\omega_{\mathrm{R}}-\omega_{\mathrm{L}}}, \quad Q_{\mathrm{R}}=-Q_{0} \frac{\omega_{0}-\omega_{\mathrm{L}}}{\omega_{\mathrm{R}}-\omega_{\mathrm{L}}}, \\
& P_{\mathrm{R}}=\omega_{0} Q_{0} \frac{\omega_{\mathrm{R}}}{\omega_{0}} \frac{\omega_{0}-\omega_{\mathrm{L}}}{\omega_{\mathrm{R}}-\omega_{\mathrm{L}}}, \quad P_{\mathrm{L}}=-\omega_{0} Q_{0} \frac{\omega_{\mathrm{L}}}{\omega_{0}} \frac{\omega_{\mathrm{R}}-\omega_{0}}{\omega_{\mathrm{R}}-\omega_{\mathrm{L}}} .
\end{aligned}
$$

The relative positioning of $\omega_{\mathrm{L}}, \omega_{0}$ and $\omega_{\mathrm{R}}$ is important. In the limit $\omega_{\mathrm{L}} \rightarrow 0, \omega_{\mathrm{R}} \rightarrow \infty, Q_{\mathrm{L}} \rightarrow Q_{0}, Q_{\mathrm{R}} \rightarrow$ $0, P_{\mathrm{R}} \rightarrow \omega_{0} Q_{0}, P_{\mathrm{L}} \rightarrow 0$. In such a case, we expect a pure Kolmogorov spectrum in the right window of transparency because neither of the thermodynamic parameters $T$ or $\mu$ will be important there. Indeed, because $E_{\omega} \rightarrow 0$ as $\omega \rightarrow \infty$, we expect $T=0$. On the other hand, for $\omega_{\mathrm{L}} \leq \omega_{0} \ll \omega_{\mathrm{R}}$, i.e. when $\omega_{\mathrm{R}} \rightarrow \infty, Q_{\mathrm{L}} / Q_{0} \rightarrow$ $1, Q_{\mathrm{R}} / Q_{0} \rightarrow 0, P_{\mathrm{R}} / P_{0} \rightarrow 1-\omega_{\mathrm{L}} / \omega_{0}$ and $P_{\mathrm{L}} / P_{0}=-\omega_{\mathrm{L}} / \omega_{0}$. Observe that, whereas in this limit all particles travel to small $\omega$, the amount of energy traveling to low frequencies depends on the relative positions of source at $\omega_{0}$ and sink at $\omega_{\mathrm{L}}$. If $\omega_{\mathrm{L}} / \omega_{0} \rightarrow 0$, then all energy goes to high frequencies. But there are circumstances, e.g. semiconductor lasers $[40,45]$, where $\omega_{0}$ and $\omega_{\mathrm{L}}$ may be close and then a significant amount of energy may be absorbed by the low frequency sink. In this case, we would expect a mixed spectrum in the left window of transparency which depends on all four constants, $P, Q, T$ and $\mu$. In general, then, we expect that the stationary attractor of (3.6) will be the four-parameter family

$$
n_{k}=n_{k}(T, \mu, P, Q)
$$

with the parameters $T, \mu, P$ and $Q$ determined by the total energy $E$, total particle number $N$ and the rates of pumping $Q_{0}$ and $P_{0}$ by the source. Even if $P_{\mathrm{L}}=0$, the spectrum in the left window may very well depend on $Q$ and the thermodynamic parameters $T$ and $\mu$.

This richer solution family can be readily seen by the so called "differential" or Fokker-Planck (FP) approximation of the collision integral [21]. Suppose that the integrand in (3.16) has its principal support near $\omega_{1}=\omega_{2}=\omega_{3}=\omega$, then we can write (3.16) as [32]

$$
\frac{\partial N_{\omega}}{\partial t}=\frac{\partial^{2} K}{\partial \omega^{2}},
$$

where $K[n]=S_{0} \omega^{(\sigma+6)} n^{4}\left(\partial^{2} / \partial \omega^{2}\right)(1 / n)$ and $S_{0}$ is a calculable constant. From (3.22), we see that the stationary solutions of (3.22) are given by solving

$$
K[n]=S_{0} \omega^{(\sigma+6)} n^{4} \frac{\partial^{2}}{\partial \omega^{2}} \frac{1}{n}=Q \omega+P,
$$

whose general solution will depend on four parameters, $Q, P$, and the two constants of integration, which we call $T$ and $\mu$, associated with $\left(\partial^{2} / \partial \omega^{2}\right)(1 / n)$. As an exercise, show that all special solutions (3.13) (take $Q=P=0$ ), (3.18) (take $P=0$; seek solution $n_{k}=c_{1} Q^{1 / 3} \omega^{-x}$ ), and (3.19) (take $Q=0$; seek solution $n_{k} c_{2} P^{1 / 3} \omega^{-x}$ ) satisfy (3.23). Similar reductions [33,40], $K_{\text {boson }}=S_{0} \omega^{(\sigma+6)}\left(n^{4}\left(\partial^{2} / \partial \omega^{2}\right)(1 / n)-n^{2}\left(\partial^{2} / \partial \omega^{2}\right) \ln n\right), K_{\text {fermion }}=$ $S_{0} \omega^{(\sigma+6)}\left(-n^{4}\left(\partial^{2} / \partial \omega^{2}\right)(1 / n)-n^{2}\left(\partial^{2} / \partial \omega^{2}\right) \ln n\right)$, obtain for bosons or fermions. Note that there are no power law solutions near $\omega=\infty$ for either bosons or fermions (contrary to what is claimed [33]). We emphasize, however, 
that the finite flux solutions for boson and fermion fields in non-equilibrium situations will be very important. As an example, in a recent paper [40,45], we have shown how finite flux carrier distributions in semiconductor lasers may enhance efficiency. There seems to be very little recognition in the literature of the fact that the quantum Boltzmann equation has a much richer class of stationary solutions than the Fermi-Dirac spectrum.

The capacity of the pure Kolmogorov solutions (3.18) and (3.19) may be found by examining the convergence of (3.18) near $k=0$, and (3.19) near $k=\infty$. For (3.18),

$$
\int_{0} n_{k} \mathrm{~d} \vec{k}=c_{1} Q^{1 / 3} \int_{0} k^{-(2 \gamma / 3)+\alpha / 3} \frac{\mathrm{d} k}{k},
$$

which converges for $\alpha>2 \gamma$ (finite capacity) and diverges for $\alpha \leq 2 \gamma$ (infinite capacity). For example, for water waves where $\gamma=3, \alpha=\frac{1}{2}$, the origin $k=0$ has an infinite capacity to absorb "particles" (i.e. longer and longer waves), whereas for optical waves of diffraction and superfluids (nonlinear Schrödinger; $\alpha=2, \gamma=0$ ), the origin $k=0$ has finite capacity. We will see in Section 4 that, in the latter case, fully nonlinear condensates and filaments then become important. The capacity of (3.19) is determined by examining the convergence or divergence of

$$
\int^{\infty} \omega_{k} n_{k} \mathrm{~d} \vec{k}=c_{2} P^{1 / 3} \int^{\infty} k^{-(2 \gamma / 3)+\alpha} \frac{\mathrm{d} k}{k}
$$

near $k=\infty$. For $3 \alpha \geq 2 \gamma$, there is infinite capacity; for $3 \alpha<2 \gamma$, there is finite capacity. In the latter case (as in the case for gravity water waves), one needs some sink at large $k$ to absorb the energy and particle throughput. For moderate flux ratio $P$, this is achieved for gravity waves by the onset of surface tension dominated three-wave resonances at wavelengths of about $1 \mathrm{~cm}$ and from there the energy is carried to a viscous sink at much smaller wavelengths. For stronger flux rates, white caps can form [48].

The validity of the weak turbulence approximation of separation of scales can also be evaluated on the pure finite flux spectra (3.18) and (3.19). For (3.18),

$$
\frac{t_{\mathrm{L}}}{t_{\mathrm{NL}}}=\frac{1}{\omega_{k} n_{k}} \frac{\partial n_{k}}{\partial t}=c \varepsilon^{4} Q^{2 / 3} k^{(2 \gamma-4 \alpha) / 3}
$$

and for (3.19)

$$
\frac{t_{\mathrm{L}}}{t_{\mathrm{NL}}}=c \varepsilon^{4} P^{2 / 3} k^{(2 \gamma / 3)-2 \alpha}
$$

We see $t_{\mathrm{L}} / t_{\mathrm{NL}}$ diverges at $k=\infty$ (0) for (2.28) when $\beta>2 \alpha(\beta<2 \alpha)$, at $k=0(\infty)$ for (3.18) when $\gamma<$ $2 \alpha(\gamma>2 \alpha)$, and at $k=\infty(0)$ when $\gamma>3 \alpha(\gamma<3 \alpha)$ for (3.19). In each case, we can define the scale at which the wave turbulence approximation fails. For the three-wave energy flux spectrum (2.28), it fails when $k>k_{\mathrm{NL}}=\exp ((1 / 2(\beta-2 \alpha))(\ln (1 / P)))$ and $\beta>2 \alpha$ (in what follows we absorb the amplitude parameter $\varepsilon$ into the fluxes $P$ and $Q$ ) or when $k<k_{\mathrm{NL}}=\exp (-(1 / 2(2 \alpha-\beta))(\ln (1 / P)))$ and $\beta<2 \alpha$. For the four-wave energy flux spectrum (3.19), it fails when $k>k_{\mathrm{NL}}=\exp ((1 /(\gamma-3 \alpha)) \ln (1 / P))$ and $\gamma>3 \alpha$ or when $k<$ $k_{\mathrm{NL}}=\exp ((-1 /(3 \alpha-\gamma))(\ln (1 / P)))$ and $\gamma<3 \alpha$. For the four-wave particle flux spectrum (3.18), it fails when $k<k_{\mathrm{NL}}=\exp ((-1 /(2 \alpha-\gamma)) \ln (1 / Q))$ and $\gamma<2 \alpha$ and when $k>k_{\mathrm{NL}}=\exp ((1 /(\gamma-2 \alpha))(\ln (1 / Q)))$ and $\gamma>2 \alpha$. Since in all cases $P$ and $Q$ are small, the failure for the energy flux spectral (2.28) and (3.19) occurs at large $k$ when $\beta>2 \alpha$ and $\gamma>3 \alpha$. In this case, $k_{\mathrm{NL}}$ should be compared with the dissipation wavenumber $k_{\mathrm{d}}$ or another large wavenumber where new behavior occurs such as is the case, e.g. at the transition between gravity-dominated and surface tension-dominated water waves. When $\beta<2 \alpha$ and $\gamma<3 \alpha$, the failure occurs at small $k$ and then $k_{\mathrm{NL}}$ should be compared against the forcing wavenumber $k_{\mathrm{f}}$ at which energy is injected. In the limits $k_{\mathrm{f}} \rightarrow 0$ and $k_{\mathrm{d}} \rightarrow \infty$, there is always a range of wavenumber where wave turbulence fails unless $\beta=2 \alpha$ or $\gamma=3 \alpha$. The critical scale $k_{\mathrm{NL}}$ for the particle flux spectrum should also be compared with the forcing wavenumber $k_{\mathrm{f}}$ (now considered 
large) and a possible sink $k_{\mathrm{d}}$ near $k=0$ as would be the case, e.g. when lasing occurs in semiconductors. In the next section, we shall argue that the breakdown at small $k$ is connected with the building of condensates and the breakdown at large $k$ is connected with the formations of shocks in some derivative of the field variable. We give two examples, but cannot say rigorously if they are typical of some universal behavior or simply special cases.

In the limit of small negative $\gamma-3 \alpha$, Gurarie [50] has calculated a renormalized KZ exponent for four-wave processes. He obtains the value $\frac{2}{3} \gamma+d-\frac{2}{9}(\gamma-3 \alpha)$ for the corrected exponent. We have not been able to find a physical understanding of this result by introducing a small condensate at $k=0$ (which is the most likely physical manifestation of the breakdown) and then recasting the problem in transformed variables. We, therefore, do not yet know what the Gurarie result might mean. It would appear that the flux connected with such a solution is not constant in $k$.

\section{Breakdown of the weak turbulence approximation and the onset of intermittency}

We have already seen the warning signs. The fact that, on the pure Kolmogorov spectra (2.28), (3.18) and (3.19), the ratios of the local linear to nonlinear time scales $\left(\varepsilon^{2} P^{1 / 2} k^{(\beta-2 \alpha)}, \varepsilon^{4} Q^{2 / 3} k^{2 / 3(\gamma-2 \alpha)}, \varepsilon^{4} P^{2 / 3} k^{2 / 3(\gamma-3 \alpha)}\right)$ can diverge at both $k=\infty$ (for $\beta>2 \alpha, \gamma>2 \alpha, \gamma>3 \alpha$, respectively) and at $k=0$ (for $\beta<2 \alpha, \gamma<2 \alpha, \gamma<3 \alpha$, respectively) suggests that fully nonlinear processes, ignored in the weak turbulence approximation, may be relevant. This is on top of the fact that, again on the pure Kolmogorov spectra (2.28), (3.18) and (3.9), these attracting solutions may only be able to absorb a finite amount of energy or particles. This finite capacity suggests that, after a finite time $t^{*}$ when these spectra have absorbed all they can, the Holder exponents of field differences may be less than 1 and the fields themselves have a fractal nature, and it is not only the pure Kolmogorov solutions which bring about the breakdown. Although more complicated to work out, it is clear that the general finite flux solutions of (2.26) (function of $T$ and $P$ ) and (3.6) (function of $T, \mu, P, Q$ ) can also bring energy and/or particles to regions of $k$ space where the ratios of nonlinear terms to linear terms in the original PDE are no longer small. For example, in systems of nonlinear Schrödinger type (and this includes a wide range of systems from superfluids to optical waves of diffraction to the carrier distributions in semiconductor lasers) with Hamiltonian (3.4), it is clear that if $T \sim k^{\gamma}$ and $\omega_{k} \sim k^{\alpha}$ the ratios of nonlinear to linear terms become unbounded for small $k$ if $\alpha$ exceeds some function (depending on how the amplitude $A_{k}$ scales) of $\gamma$.

We note here that (2.2) has the scaling symmetry

$$
A^{s}(\vec{k}, t)=\lambda^{b} B^{s}\left(\vec{K}=\lambda \vec{k}, T=\lambda^{-\alpha} t\right),
$$

if $b=\beta+d-\alpha=\gamma+d-\beta$. It will turn out that this scaling symmetry is only preserved by the long-time statistics of weak turbulence for small scales when $\beta=2 \alpha$ and $\gamma=3 \alpha$, or for large scales when $\gamma=2 \alpha$. It is noteworthy that, in general, weak turbulence does not evolve to solutions which preserve the scaling symmetries of the original system. In the Kolmogorov' 41 theory of hydrodynamic turbulence, scaling symmetry is preserved whereas, as we have already pointed out, the true behavior of statistical hydrodynamics leads to solutions which break that symmetry and for which the ratios of the structure functions $S_{n} /\left(S_{2}\right)^{n / 2}$ diverge at small scales.

It is, therefore, relevant to examine the long-time behavior of the structure functions

$$
S_{N}(\vec{r})=\left\langle\left(v^{s}(\vec{x}+\vec{r})-v^{s}(\vec{x})\right)^{N}\right\rangle
$$

for weak turbulence for small $r=|\vec{r}|$. While we will give a fuller report elsewhere, here we will give some of the main results. In particular, we will evaluate $S_{N}(\vec{r})$ on the finite energy flux spectra using the field $v^{s}(\vec{x})$ whose pair correlation function $R_{v}^{s s^{\prime}}(\vec{r})=\left\langle v^{s}(\vec{x}) v^{s^{\prime}}(\vec{x}+\vec{r})\right\rangle$ has the spectral energy density as its Fourier transform. We will be interested mainly in those cases where the structure functions are universal, namely are dominated at small scales $r$ by the universal spectra (2.28) and (3.19) in the window of transparency and not by the non-universal part of $n_{k}$ 
outside of this window. The uniformity of the ratio $S_{N}$ to $\left(S_{2}\right)^{N / 2}$ as $r \rightarrow 0$ will give a measure of how close to joint Gaussianity the system remains.

On the particle flux spectra dominated by low $k$ (large $r$ ) behavior, the structure functions are no longer the relevant objects. In that case, we calculate the cumulants $R^{(N)}$ directly and, as a measure of joint Gaussianity, see how the ratio of $R^{(N)}$ to $\left(R^{(2)}\right)^{N / 2}$ behaves in the large $r$ limit. In this case, the relevant physical field is $u^{s}(\vec{x})$, defined earlier, as its pair correlation function has the particle density $n_{k}$ as its Fourier transform.

We begin by examining $S_{N}(\vec{r})$ on (2.28). For convenience, we omit the sign parameter $s$.

$$
S_{2}(r)=\left\langle(v(x+r)-v(x))^{2}\right\rangle=2\left(R_{v}^{(2)}(0)-R_{v}^{(2)}(r)\right)=2 \int \omega_{k} n_{\vec{k}}\left(1-\mathrm{e}^{\mathrm{i} \vec{k} \cdot \vec{r}}\right) \mathrm{d} \vec{k} .
$$

If $n_{k}$ is isotropic, then the bracket in the integrand can be replaced by $(1-\cos \vec{k} \cdot \vec{r})$ which is of order $(k r)^{2}$ for small $k r$. This simply reflects the fact that, for the ensemble of initial fields, velocity differences should be smooth (i.e. differentiable) and the structure function $S_{N}(r)$ proportional to $r^{N}$. In general, this property will fail to hold for long times because of the algebraic dependence (2.28) of the spectral densities on $k$. From (2.28), $\omega_{k} n_{k} \sim k^{(\alpha-\beta-d)}$ in some window $K_{\mathrm{I}}<k<K_{\mathrm{U}}$ where $K_{\mathrm{I}}$ and $K_{\mathrm{U}}$ are the infrared and ultraviolet cutoffs, respectively. For $\beta-\alpha>0$, the finite capacity case, we can take the ultraviolet cutoff $K_{\mathrm{U}}$ to be $\infty$. For $k<K_{\mathrm{I}}$, the infrared cutoff, the spectrum is non-universal. However, when we evaluate $\lim _{K_{\mathrm{I}} \rightarrow 0} \int \omega_{k} n_{\vec{k}}(1-\cos \vec{k} \cdot \vec{r}) \mathrm{d} \vec{k}$ on the spectrum (2.28), we find that we can take the limit when $\beta-\alpha<2$ and then $S_{2}(r) \sim P^{1 / 2} r^{(\beta-\alpha)}$. The non-universal contribution will behave as $r^{2}$ which, since $\beta-\alpha<2$, is less than $r^{(\beta-\alpha)}$ for small $r$. Observe that this suggests that in the long-time limit, the members of the ensemble of field differences $v(x+r)-v(x)$ are not differentiable but only Holder continuous with "average" Holder exponent $\frac{1}{2}(\beta-\alpha)$. Frisch [37] discusses relations between the exponent $\xi_{N}$ of the $N$ th-order structure function and the Hausdorff dimension of singularity sets. In the present work, therefore, we are principally interested in parameter values $\alpha, \beta$ where $0<\beta-\alpha<2$. For $2 \alpha>\beta>\alpha$, we expect that the shape of the signal $v(x)$ is monofractal. For $\beta>2 \alpha$, the signal will also contain intermittent behavior.

Next, we evaluate $S_{3}(r)=3\left(R_{v}^{(3)}(0, r)-R_{v}^{(3)}(0,-r)\right)$ is the small $r$ limit. The leading-order behavior vanishes for two reasons. First, $Q_{0}^{(3)}$ contains a fast oscillation which means its Fourier transform $R_{0}^{(3)}$ will decay as $\omega_{0} t \rightarrow$ $\infty$. Moreover, $Q_{0}^{(3)}$ itself decays on the long-time scale $\varepsilon^{2} \omega_{0} t \rightarrow \infty$. The principal surviving contribution to $S_{3}(r)$ comes from $\omega^{3 / 2} \tilde{Q}_{1}^{(3)}$, where $\tilde{Q}_{1}^{(3)}$ is given by (2.17). Remember, we are defining the structure functions in terms of the field $v(\vec{x})$ whose Fourier transform is $\omega^{1 / 2} A_{\vec{k}}$. Taking $n_{k}$ to be given by (2.28), $\omega^{3 / 2} \tilde{Q}_{1}^{(3)}$ behaves as $k^{3 \alpha / 2} \cdot k^{\beta} P k^{-2(\beta+d)} \cdot k^{-\alpha}$. The term $\mathrm{e}^{\mathrm{i} \vec{k} \cdot \vec{r}}-\mathrm{e}^{-\mathrm{i} \vec{k} \cdot \vec{r}}$ behaves as $(k r)^{3}$ as the $k r$ contribution vanishes on integration. The integration over $\mathrm{d} \vec{k} \cdot \mathrm{d} \vec{k}^{\prime}$ behaves as $k^{(2 d-1)} \mathrm{d} k \mathrm{~d} u$ (write $k^{\prime}=k u$ ). Thus, convergence as $K_{\mathrm{I}} \rightarrow 0$ is guaranteed because if $0<\beta-\alpha<2$, then $\beta-\frac{1}{2} \alpha<3$ as long as $\alpha \leq 2$. We thus require $0<\alpha \leq 2$. Then $S_{3}(r) \sim \varepsilon P r^{\beta-\alpha / 2}$. Similar reasoning leads to $S_{4}(r)=3 S_{2}^{2}(r)+2 R_{v}(0,0,0)-4 R_{v}(0,0, r)+6 R_{v}(0, r, r)-4 R_{v}(0,0,-r)$ behaving as $3 \operatorname{Pr}^{(2 \beta-2 \alpha)}\left(1+\varepsilon^{2} P^{1 / 2} r^{(2 \alpha-\beta)}\right)$. Likewise, the ratio of $S_{N}(r)$ to $\left(S_{2}\right)^{N / 2}$, for $N$ even, can be written as a constant times a finite series $1+\sum_{s=1}^{(N / 2)-1} C_{N s}\left(P^{1 / 2} r^{(2 \alpha-\beta)}\right)^{s}$, where we have now absorbed the $\varepsilon$ factor into $P$. The coefficients $C_{N s}$ diverge with $N$. The series becomes non-uniform as $r \rightarrow 0$ and, therefore, the statistics deviates significantly from joint Gaussian when $\beta>2 \alpha$. We find, therefore, that $S_{N}(\vec{r})$ is dominated by the universal part of the $n_{k}$ spectrum in the parallelogram $0<\alpha \leq 2,0<\beta-\alpha<2$ for which the diagonal is $\beta=2 \alpha$. On one side $\beta>2 \alpha$, joint Gaussianity fails at small scales; on the other, $\beta<2 \alpha$, it fails at large scales.

For four-wave processes, a similar situation obtains. There, convergence of $S_{2}(r)$ as $r \rightarrow 0$ required $0<\frac{2}{3} \gamma-\alpha<$ 2. If this holds, and $0<\alpha \leq 2$, all later $S_{N}$ converge as $K_{\mathrm{I}} \rightarrow 0$ and are therefore dominated (as $r \rightarrow 0$ ) by the universal part of the $n_{k}$ spectrum. We find that for even $N$, the ratio of $S_{N}(r)$ to $\left(S_{2}\right)^{N / 2}$ is proportional to $1+\sum_{s=1}^{(N / 2)-1} C_{N s}\left(P^{1 / 3} r^{\alpha-\gamma / 3}\right)^{s}$. Again, the coefficients $C_{N s}$ diverge with $N$. On the particle flux spectrum (3.19), we find that for large $r$, the ratio $R^{(N)}$ to $\left(R^{(2)}\right)^{N / 2}$ diverges as $C_{N}\left(Q^{1 / 3} r^{(2 \alpha-\gamma) / 3}\right)^{(N / 2)-1}$. 
For all cases, the precise dependence of $C_{N s}$ on $N$ has yet to be determined. From a knowledge of its behavior, we could infer the behavior of the PDF for the field differences in the limit as $r \rightarrow 0$.

Note that the original scaling property of $(2.2), A^{s}(\vec{k}, t)=\lambda^{b} B^{s}\left(\vec{K}=\lambda \vec{k}, T=\lambda^{-\alpha} t\right), b=\beta+d-\alpha=\gamma+d-\beta$ is only preserved by the long-time statistics on the energy flux spectrum when $\beta=2 \alpha, \gamma=3 \alpha,(b=\alpha+d)$ and on the particle flux spectrum when $\gamma=2 a\left(b=\frac{1}{2} \alpha+d\right)$.

What we have demonstrated that for almost all values of the parameters $\alpha, \beta, \gamma$, the long-time solutions to the kinetic equations for wave turbulence lead to the invalidation of the premises ((i) $t_{\mathrm{L}} / t_{\mathrm{NL}}$ uniformly small, and (ii) close to joint Gaussian behavior) on which the theory is founded. But this is not a catastrophe. On the contrary, it affords an opportunity to develop a theory which may well act as a paradigm for many other turbulent fields. The reasons for this optimism is that, in the wave turbulence situation, one can identify in many cases the strongly nonlinear and coherent fluctuations that occur. We now discuss two applications.

Currently, we are attempting to develop a combined wave turbulence and sparse large fluctuations picture for optical turbulence. This situation has been described by us previously $[32,35]$ so we will only briefly outline the ideas here. The model is the nonlinear Schrödinger equation

$$
u_{t}+\mathrm{i} \nabla^{2} u+\mathrm{i} a u^{2} u^{*}=0
$$

for which $\omega_{k}=k^{2}$ and $T_{k k_{1}, k_{2} k_{3}}=-a$. Let us imagine that we insert particles and energy in a range of wavenumbers where the weak turbulence approximation is valid. Four-wave resonances serve to redistribute particles and energy with most energy (some particles) going to high $k$ and most particles (some energy) going to lower $k$. This redistribution is independent of the sign of $a$. Because the origin $k=0$ has only finite capacity, once the particles reach $k=0$, a condensate will begin to grow. In the defocusing case $a<0$, patches of condensate with different phases will be built in different spatial domains and small fluctuations will propagate as dispersive waves on top of these condensates. The dispersive waves will have a new dispersion relation discussed in Example (a) of Section 2.1. The domain walls will also interact. For the focusing case $a>0$, a more dramatic chain of events occurs. Condensates (and indeed long periodic waves) are modulationally unstable. From these modulational instabilities, collapsing filaments are formed giving rise to a very fast (the inviscid NLS equation for $d \geq 2$ has a finite time singularity) transfer of particles (the energy, i.e. $\int\left(|\nabla u|^{2}-\frac{1}{2} a|u|^{4}\right) \mathrm{d} \vec{x}$ of each is zero) from $k=0$ to $\infty$. The collapses occur randomly in space and time. Their average frequency of occurrence would appear to be proportional to $Q_{\mathrm{L}}$, the rate at which particles flow towards the origin. This transfer of particles is carried by organized and coherent structures, and very different to the direct cascade of particles due to four-wave resonances. As the collapsing filaments reach $k=\infty$, the presence of dissipation causes incomplete burnout because each collapsing filament (in 2D) only carries exactly the critical number $N_{\mathrm{c}}$ of particles requires to sustain the collapse. As a result, the collapse is arrested and only $15-20 \%$ of the particle number $N_{\mathrm{c}}$ is lost. Nevertheless, significant particle loss occurs and this will produce large fluctuations (spikes) in the dissipation signal, each spike coinciding precisely with a collapse event. Since the energy is non-positive definite, the transport of particles to high $k$ can lead to an energy source at large $k$. Specifically, the viscous sink at large $k$ will absorb a portion of the potential energy component $-(a / 2) \int|u|^{4} \mathrm{~d} \vec{x}$ of the zero energy collapsing filament. The corresponding amount of kinetic energy component $\int|\nabla u|^{2} \mathrm{~d} \vec{x}$ is then free to be returned to the wave field. The returning particles (if $f$ is the fraction lost) build up the inverse particle flux rate from the initial input rate to $(1 / f) Q_{0}$ so that eventually all particle dissipation occurs by their transfer to $k=0$ by four-wave processes and then to $k=\infty$ by collapses.

We call this series of events the cycle of intermittency. It is very clear from our numerical experiments reported in [32] that the intermittency seen in the signal is due to these large fluctuation events. They can be suppressed by inserting damping near $k=0$. As this damping is decreased, they become more and more frequent and, at zero damping, they contribute significantly to the particle dissipation rate. Currently, we are attempting to build a consistent model of a two species gas with waves and filaments in order to describe this cycle analytically. One has a 
clear advantage in that individual collapse events, once nucleated, do not feel the waves (the time scale is too short) and that the waves feel the collapses only as a source of new waves arising from the incomplete burnout process.

The second application is deep-water gravity waves for which $\alpha=\frac{1}{2}, \beta=\frac{7}{4}, \gamma=3=2 \beta-\alpha$ so that $L_{\vec{k}, \vec{k}_{1}, \vec{k}_{2}, \vec{k}_{3}}^{S S_{1} S_{2} s_{3}}$ and $\left(1 / \omega_{k}\right)\left(L_{\vec{k} \vec{k}_{1} \vec{k}_{2}}^{S S_{1} s_{2}}\right)^{2}$ have the same degree of homogeneity. The ratio $t_{\mathrm{L}} / t_{\mathrm{NL}}$ is $P^{2 / 3} k / g$ where we have incorporated $\varepsilon$ in $P$. If this becomes of order 1 at values of $k$ less than $k_{0}=\sqrt{\rho_{\mathrm{w}} g / S}$ ( $\rho_{\mathrm{w}}$ is the water density, $g$ the gravity, $S$ the surface tension) at which surface tension effects can absorb the energy flux, then one must expect fully nonlinear behavior. This criterion, namely that $P^{2 / 3}>g / k_{0}$ translates to a criterion on the wind speed $V$ $\left(P^{2 / 3}=\left(\rho_{a} / \rho_{\mathrm{W}}\right) V^{2}, \rho_{a}\right.$ is the air density) namely $V>\left(\rho_{\mathrm{w}} / \rho_{\mathrm{a}}\right)^{1 / 2}\left(S g / \rho_{\mathrm{w}}\right)^{1 / 4}$, or approximately $6 \mathrm{~m} / \mathrm{s}$, the speed at which whitecapping first occurs. Therefore, it is likely that the coherent nonlinear structure in this case is the local formation of slope discontinuities on the water surface. A sea full of such discontinuities would lead to a Phillip's spectrum $E_{k} \sim k^{-4}$ for the energy density. It is interesting that the criterion that $P^{2 / 3}>g / k_{0}$ is also the criterion for the Kolmogorov-Zakharov spectrum (3.19) to meet the Phillip's spectrum before $k=k_{0}$ [48].

\section{How the Kolmogorov spectrum is realized: a new anomaly}

Previous thinking [32,47] as to how the Kolmogorov spectra (2.28), (3.18) and (3.19) are reached may not be generally correct in the finite capacity cases. The idea was that an initial isotropic spectrum on compact support in $k$-space would propagate to $k=\infty$ (or $k=0$ ) as a front $k=k_{\mathrm{f}}(t)$ for which $n_{k} \rightarrow 0$ for $k>k_{\mathrm{f}}(t)$ and $n_{k} \rightarrow c P^{1 / 2} k^{-(\beta+d)}$ for $k<k_{\mathrm{f}}(t)$. Namely, the front propagates so as to leave the stationary Kolmogorov solution in its wake. The transition would be described as a self-similar solution of the kinetic equation

$$
n(\vec{k}, t)=\frac{1}{t^{a}} n_{0}\left(\frac{k}{t^{b}}\right) .
$$

Substitution of (5.1) into the kinetic equation (5.2) gives an integral equation for $n_{0}\left(\eta=k / t_{b}\right)$ and one relation

$$
a=1+b(2 \beta+d-a)
$$

between $a$ and $b$. For infinite capacity case, one can obtain a second relation by realizing that eventually all the energy put into the system will reside in the wake of the self-similar solution. Assuming that the rate of energy input is constant, we have that $\int \omega_{k} \vec{n}_{k} \mathrm{~d} \vec{k}$ grows like $t$ and this means that

$$
a+1=b(\beta+d) .
$$

Together (5.2) and (5.3) give

$$
b=\frac{1}{\alpha-\beta}, \quad a=\frac{\beta+d}{\alpha-\beta},
$$

which for, $\alpha>\beta$, are both positive. The front moves towards infinity at the finite rate $k=k_{\mathrm{f}}(t)=t^{1 /(\alpha-\beta)}$. In the wake, $n(\vec{k}, t) \rightarrow c P^{1 / 2} k^{-(\beta+d)}$ since $a=(\beta+d) b$.

This is, e.g. the case for water waves near $k=0$ (with an analogous result from the four-wave resonance kinetic equation) and for NLS near $k=\infty$.

But, for the finite capacity case $\beta>\alpha$ and $b<0$. It is useful in this case to write (5.1) as

$$
n_{k}(\vec{k}, t)=\frac{1}{\tau^{a}} n_{0}\left(\frac{k}{\tau^{b}}\right) \quad \text { with } \tau=t^{*}-t .
$$

Again we find (5.2) obtains. However, we cannot assume (5.3) because in the finite capacity case the energy contained in the Kolmogorov solution cannot grow past its finite capacity. Because $b<0$, the front will reach $k=\infty$ in a 
finite time $t^{*}$. For $0<t<t^{*}$, a second relation between $a$ and $b$ must be found by treating the nonlinear integral equation for $n_{0}\left(\eta=k\left(t^{*}-t\right)^{-b}\right)$ as a nonlinear eigenvalue problem. Assuming that the wake approaches a power law state $k^{-\sigma}$ means that $a=b \sigma$. But $a / b=\sigma$ may not be $(\beta+d)$, the Kolmogorov power law.

We now have two examples where this is not the case. Both are reported in this volume, one here and one in [53]. For three-wave processes, the first to realize this possibility were Galtier et al. [46,49] for the case of weak MHD turbulence dominated by Alfven waves. This is consistent with the observations of Frisch and Fournier [51] on the inviscid Burgers equation. More recently, Svistunov [52] and more recently Pomeau and Rica [53] have noted similar behavior in the building of Bose condensates (cf. NLS). What we found in the MHD context was the following. For $0<t<t^{*}$, the wake solution was $n(\vec{k}, t) \sim k^{-7 / 3}$ which is steeper than $k^{-2}$, the Kolmogorov spectrum. However, as $t$ approached $t^{*}$, a remarkable transition took place. For $t \geq t^{*}$, disturbances beginning at large $k$ propagated back along the $k^{-7 / 3}$ spectrum and lifted it to the $k^{-2}$ shape. It would appear that once the connection to $k=\infty$ is made, the circuit between source and sink is closed allowing the usual finite flux spectrum to be set up.

This remarkable behavior resembles to some extent the behavior observed in critically self-organized systems. Can it be that in finite capacity cases, the Kolmogorov spectrum of wave turbulence is indeed such a "critical" state?

\section{Acknowledgements}

The authors thank Oleg Zaboronski for numerous helpful discussions. We are also grateful for support from EC Contract FMRX-CT98-0175 and from NSF Grant DMS 0072803.

\section{References}

[1] K. Hasselmann, On the nonlinear energy transfer in a gravity-wave spectrum I, J. Fluid Mech. 12 (1962) $481-500$.

[2] K. Hasselmann, On the nonlinear energy transfer in a gravity-wave spectrum II, J. Fluid Mech. 15 (1963) $273-281$.

[3] K. Kenyon, Discussion, Proc. Roy. Soc. London A 299 (1456) (1967) 141-144.

[4] D.J. Benney, P.G. Saffman, Nonlinear interactions of random waves in a dispersive medium, Proc. Roy. Soc. London A 289 (1966) 301-320.

[5] D.J. Benney, A.C. Newell, Sequential time closures of interacting random waves, J. Math. Phys. 46 (1967) 363.

[6] D.J. Benney, A.C. Newell, Statistical Properties of the Sea, Physics of Fluids Special Issue, Kyoto Meeting of International Union of Theoretical and Applied Mechanics, September 1966, Vol. 10, 1967, S281 pp.

[7] V.E. Zakharov, N.N. Filonenko, Energy spectrum for stochastic oscillations of the surface of a fluid, Dokl. Akad. Nauk SSSR 170 (6) (1966) 1292-1295 [English transl. in Sov. Math. Dokl.].

[8] V.E. Zakharov, N.N. Filonenko, Weak turbulence of capillary waves, Zh. Prikl. Mekh. I Tekn. Fiz. (5) (1967) 62-67 [English transl. in J. Appl. Mech. Tech. Phys.].

[9] V.E. Zakharov, Weak turbulence in media with decay dispersion law, Zh. Prikl. Mekh I Tekhn. Fiz. 4 (1965) 35 [English transl. in J. Appl. Mech. Tech. Phys.].

[10] V.E. Zakharov, On the spectrum of turbulence in plasma without magnetic field, Zh. Eksper. Teoret. Fiz. 51 (1966) 686-696 [English transl. in Sov. Phys. JETP 24 (1967) 455-459].

[11] A.C. Newell, The closure problem in a system of random gravity waves, Rev. Geophys. 6 (1968) 1-31.

[12] D.J. Benney, A.C. Newell, Random wave closures, Stud. Appl. Math. 48 (1) (1969) 29-53.

[13] A.C. Newell, P.J. Aucoin, Semidispersive wave systems, J. Fluid Mech. 49 (1971) 593-609.

[14] V.E. Zakharov, R.Z. Sagdeev, On spectra of acoustic turbulence, Dokl. Akad. Nauk SSSR 192 (2) (1970) $297-299$ [English transl. in Sov. Phys. JETP 35 (1972) 310-314].

[15] E.A. Kuznetsov, On turbulence of ion sound in plasma in a magnetic field, Zh. Eksper. Teoret. Fiz. 62 (2) (1972) 584-592 [English transl. in Sov. Phys. JETP 35 (1972) 310-314].

[16] V.E. Zakharov, S.L. Musher, On Kolmogorov spectra in a system of nonlinear oscillators, Dokl. Akad. Nauk SSSR 209 (5) (1973) 1063-1065 [English transl. in Sov. Phys. Dokl. 18 (1973)].

[17] A. Hasegawa, K. Mima, Pseudo-three-dimensional turbulence in magnetized non-uniform plasma, Phys. Fluids 21 (1978) 87.

[18] E.N. Pelinovskii, Wave turbulence on a beta-plane, Okeanologia 18 (2) (1978) 192-195 (in Russian). 
[19] D.R. Crawford, P.G. Saffman, H.C. Yuen, Evolution of a random inhomogeneous field of nonlinear deep-water gravity waves, Wave Motion $2(1980) 1-16$.

[20] R. Peierls, Quantum Theory of Solids, Clarendon Press, Oxford, 1955/Pergamon Press, Oxford, 1981.

[21] S. Hasselmann, K. Hasselmann, J.H. Allender, T.P. Barnett, J. Phys. Oceanogr. 15 (1985) 1378.

[22] V.S. L'vov, G.E. Falkovich, On anisotropic spectra of weak and sound turbulence, Zh. Eksper. Teoret. Fiz. 80 (2) (1981) $593-596$ [English transl. in Sov. Phys. JETP 53 (1981) 200-300].

[23] R.S. Iroshnikov, Possibility of formation of a non-isotropic spectrum of wind waves by their weak nonlinear interaction, Dokl. Akad. Nauk SSSR 280 (6) (1985) 1321-1325 [English transl. in Sov. Phys. Dokl. 30 (1985) 126-128].

[24] Y.L. Klimontovich, D. Kremp, W.D. Kraeft, Adv. Chem. Phys. 68 (1987) 175.

[25] A.M. Balk, V.E. Zakharov, On stability of weak turbulence Kolmogorov spectra, Dokl. Akad. Nauk SSSR 299 (5) (1988) 1112-1115 [English transl. in Sov. Phys. Dokl. 33 (1988) 270-273].

[26] A.M. Balk, V.E. Zakharov, Stability of weak turbulence Kolmogorov spectra, in: Plasma Theory and Nonlinear and Turbulent Processes in Physics, Proceedings of the International Workshop, Kiev, April 1987, World Scientific, Singapore, 1988, pp. 359-376.

[27] G.E. Falkovich, A.V. Shafarenko, On the stability of Kolmogorov spectra of a weak turbulence, Physica D 27 (1987) 311-399.

[28] V.E. Zakharov, E.I. Shulman, On additional motion invariants of classical Hamiltonian wave systems, Physica D 29 (1988) $283-320$.

[29] A.M. Balk, S.V. Nazarenko, On the physical realizability of anisotropic Kolmogorov spectra of weak turbulence, Sov. Phys. JETP 70 (1990) 1031.

[30] A.M. Balk, S.V. Nazarenko, V.E. Zakharov, New invariant for drift turbulence, Phys. Lett. A 152 (5-6) (1991) 276-280.

[31] A.M. Balk, S.V. Nazarenko, V.E. Zakharov, Non-local turbulence of drift waves, Zh. Eksper. Teoret. Fiz. 98 (1990) 446-467 [English transl. in Sov. Phys. JETP 71 (1990) 249-260].

[32] S. Dyachenko, A.C. Newell, A. Pushkarev, V.E. Zakharov, Optical turbulence: weak turbulence, condensates and collapsing filaments in the nonlinear schrodinger equation, Physica D 57 (1992) 96-160.

[33] V.E. Zakharov, V.S. L’vov, G. Falkovich, Kolmogorov Spectra of Turbulence, Springer, Berlin, 1992.

[34] M.L. Banner, I.R. Young, Modeling spectral dissipation in the evolution of wind waves. I. Assessment of existing model performance, J. Phys. Oceanogr. 24 (1994) 1550-1571.

[35] A.C. Newell, V.E. Zakharov, Optical turbulence, in: P. Tabeling, O. Cardoso (Eds.), Turbulence: A Tentative Dictionary, Plenum Press, New York, 1995.

[36] A.N. Pushkarev, V.E. Zakharov, Turbulence of capillary waves, Phys. Rev. Lett. 76 (1996) 3326-3329.

[37] U. Frisch, Turbulence, Cambridge University Press, Cambridge, 1996.

[38] V. L'vov, Y.V. L’vov, A.C. Newell, V.E. Zakharov, Statistical description of acoustic turbulence, Phys. Rev. E 56 (390) (1997) 1.

[39] Y.V. L'vov, A.C. Newell, Semiconductor lasers and Kolmogorov spectra, Phys. Lett. A 235 (1997) 499-503.

[40] Y.V. L'vov, R. Binder, A.C. Newell, Quantum weak turbulence with applications to semiconductor lasers, Physica D 121 (1998) $317-343$.

[41] V.E. Zakharov (Ed.), Dispersive Nonlinear Waves and Weak Turbulence, Vol. 182, AMS Translations Series 2, American Mathematical Society, Providence, RI, 1998.

[42] A.M. Balk, E.V. Ferapontov, Invariants of wave system and web geometry, Am. Math. Soc. Transl. 182 (2) (1998) 1-20.

[43] A.M. Balk, V.E. Zakharov, Stability of weak-turbulence Kolmogorov spectra, Am. Math. Soc. Transl. 182 (2) (1998) 31-82.

[44] V.E. Zakharov, Weakly nonlinear waves on the surface of an ideal finite depth fluid, Am. Math. Soc. Transl. 182 (2) (1998) $167-197$.

[45] Y.V. L'vov, A.C. Newell, Finite flux solutions of the quantum Boltzmann equation and semiconductor lasers, Phys. Rev. Lett. 84 (2000) $18-94$.

[46] S. Galtier, S.V. Nazarenko, A.C. Newell, A. Pouquet, A weak turbulence theory for incompressible magnetohydrodynamics, in: T. Passot, P.L. Sulem (Eds.), Nonlinear MHD Waves and Turbulence, Lecture Notes in Physics, Springer, Berlin, 1999, pp. 291-330.

[47] G.E. Falkovich, A.V. Shafarenko, Non-stationary wave turbulence, J. Nonlinear Sci. 1 (1991) 452-480.

[48] A.C. Newell, V.E. Zakharov, Rough sea foam, Phys. Rev. Lett. 63 (1992) 1149.

[49] S. Galtier, S.V. Nazarenko, A.C. Newell, A. Pouquet, A weak turbulence theory for incompressible MHD, J. Plasma Phys. 63 (2000) 447-488.

[50] V. Gurarie, Probability density, diagrammatic technique and epsilon expansion in the theory of wave turbulence, Nucl. Phys. B 441 (1995) 569-594.

[51] U. Frisch, J.D. Fournier, Quelques resultats exacts pour l'equation de Burgers aleatoire, J. Phys. 39 (1978) C5-C19.

[52] B.V. Svistunov, Highly non-equilibrium Bose condensation in a weakly interacting gas, J. Moscow Phys. Soc. 1 (1991) 373.

[53] R. Lacaze, P. Lallemand, Y. Pomeau, S. Rica, Dynamical formation of a Bose-Einstein condensate, Physica D 152-153 (2001) 779-786. 